Bolm Inst. oceanogr., S Paulo, 27(2):153-190, 1978

\title{
SPECTRAL METHODS IN OCEANOGRAPHY WITH APPLICATIONS*
}

PEDRO ALBERTO MORETTIN \& AFRÂNIO RUBENS DE MESQUITA

Instituto de Matemática e Estatistica e Instituto Oceanográfico da Universidade de são Paulo

\section{SYNOPSIS}

This paper is a survey of the main spectral methods potentially useful in Oceanography. These methods are applied to the analysis of tides, seasonal variations and ocean geophysical oscillations. Further topics on the Response Method, the Maximum Entropy method and Rotary Components are briefly summarized. Examples of successful applications are presented.

\section{1 - INTRODUCTION}

This paper intends to be a survey of the main spectral methods potentially useful in oceanography. Some practical problems are discussed but they do not cover, of course, the range of all possible applications. Basically,

*- This work was partially supported by Projeto FINEP-IOUSP, Sub-Projeto no 3 - Hidrodinâmica Costeira, and Fundação de Amparo à Pesquisa do Estado de São Paulo (FAPESP).

- Paper presented at the 1977 Annual Meeting of the American Statistical Association, August 15-18, 1977, Chicago, Illinois.

PUBL. NO 425 DO INST. OCEAN. DA USP 
there are three types of phenomena that appear in physical oceanography: first, those which present known frequencies and phases and we are interested in their amplitudes; secondly, those which possess known frequencies, and the interest is to determine phase and amplitudes and finally there are those phenomena for which frequencies, phases and amplitudes are unknown. According to other classification the hidrodynamical ocean processes are assumed to be of planetary scale (sections 3 and 5 ), medium scale and microscale, eventhough there is not a precise separation between these scales. Processes of medium and micro-scale are further discussed in Mesquita \& Morettin, 1976.

The plan of the paper is as follows: in section 2 we describe some general spectral methods; in section 3 we present the fundamentals of tide analysis and results of a simple analysis of a tidal record are given. An application concerning seasonal variation is given in section 4 and in section 5 some preliminary analysis of GATE (GARP ATLANTIC TROPICAL EXPERIMENT) data is discussed.

\section{2 - GENERAL SPECTRAL METHODS}

Let $X=\{X(t, \omega): t \varepsilon T, \omega \varepsilon \Omega\}$ be a real-valued stochastic process, where for each $t \varepsilon T, X(t, \omega)$ is a random variable defined on the probability space $(\Omega, A, P)$. Usually we take $T=Z=\{0, \pm 1, \pm 2, \ldots\}$ or $T=R$, the set of real numbers . For fixed $\omega$ we have a realization, trajectory or sample function of $\mathrm{x}$. The set of all these realizations is the ensemble. A problem which arises in practical situations is the determination of the ensemble. Suppose, for example, that we are interested in measuring wave heights in a given area. If an instrument is attached to a buoy and thrown in a point of this area, we will have observations of a time series, which is part of a realization of the process. If the buoy is thrown in a different point we will observe another realization of the process. It is therefore necessary to stipulate the area in which we have some assurance that we are observing the same process. 
We will indicate by $\mu(t)=E[X(t, \omega)]$ the expected value of $x$ and by $C\left(t_{1}, t_{2}\right)$ the auto-covariance function of $x$, defined by

$$
\mu(t)=E[X(t, \omega)]=\int x d F(x, t),
$$

$$
C\left(t_{1}, t_{2}\right)=E\left\{\left[X\left(t_{1}, \omega\right)-\mu\left(t_{1}\right)\right]\left[x\left(t_{2}, \omega\right)-\mu\left(t_{2}\right)\right]\right\}
$$

respectively. Here, $F(x, t)=P(X(t) \leq x)$ is the distribution function of the random variable $x(t, \omega)$. If $t_{1}=t_{2}$ we have the variance of $x(t, \omega)$. We will omit the dependence on $\omega$ and write simply $X=\{X(t), t \varepsilon Z\}$.

$$
2.1 \text { - STATIONARY PROCESSES }
$$

Let $\mathrm{T}=\mathrm{Z}$ and suppose we have now a vector-valued stochastic process

$$
\underset{\sim}{X}(t)=\left[\begin{array}{ll}
X_{1} & (t) \\
X_{2} & (t) \\
\cdot \\
\cdot \\
x_{p} & (t)
\end{array}\right], t \varepsilon Z,
$$

with $\mathrm{p}$ real-valued components. We say that $\underset{\sim}{\mathrm{X}}(\mathrm{t})$ is weakly stationary or second-order stationary if

$$
\begin{aligned}
& \text { (i) } \mu_{j}(t)=E\left[x_{j}(t)\right]=\mu_{j}, \\
& \text { (ii) } c_{j k}(t+\tau, t)=\operatorname{Cov}\left\{x_{j}(t+\tau), x_{k}(t)\right\}=c_{j k}(\tau), \\
& \text { (iii) } E\left[x_{j}^{2}(t)\right]<\infty,
\end{aligned}
$$


for $t, \tau \in \mathrm{Z}$ and $\mathrm{j,k}=1,2, \ldots, \mathrm{p}$. Without loss of generality we assume that $\mu_{j}=0$, in such a way that the cross-covariance function $c_{j k}(\tau)$ of $x_{j}(t)$ with $\mathrm{X}_{\mathrm{k}}(\mathrm{t})$ can be written

$$
c_{j k}(\tau)=E\left[x_{j}(t+\tau) x_{k}(t)\right],
$$

for $t, \tau \in Z$ and $j, k=1, \ldots, p$. If $j=k$ we have the auto-covariance function of $x_{j}(t)$.

If we can assume that

$$
\sum_{\tau=-\infty}^{\infty}\left|C_{j k}(\tau)\right|<\infty, j, k=1, \ldots, p,
$$

then we define the second-order spectrum of $x_{j}(t)$ with $x_{k}(t)$ by

$$
f_{j k}(\lambda)=(2 \pi)^{-1} \sum_{\tau=-\infty}^{\infty} c_{j k}(\tau) e^{-i \lambda t},
$$

for $-\infty<\lambda<+\infty, j, k=1, \ldots, p$. If $j=k, f_{j j}(\lambda)$ is the (power) spectrum of $x_{j}(t)$ at frequency $\lambda$ and if $j \neq k, f_{j k}(\lambda)$ is the cross-spectrum of $x_{j}(t)$ with $x_{k}(t)$ at $\lambda$. Expression (2.5) is a mixing condition in the sense that $x_{j}(t+\tau)$ and $x_{k}(t)$ become less dependent as $|\tau| \rightarrow \infty$. $f_{j k}(\lambda)$ is bounded, uniformly continuous and of period $2 \pi$; moreover,

$$
f_{j k}(\lambda)=\overline{f_{k j}(\lambda)}=f_{k j}(-\lambda) \text {. }
$$

Since $f_{j k}(\lambda)$ is complex it can be written

$$
f_{j k}(\lambda)=\left|f_{j k}(\lambda)\right| e^{i \theta_{j k}(\lambda)}=c_{j k}(\lambda)+i q_{j k}(\lambda) .
$$


The real part of $f_{j k}(\lambda), c_{j k}(\lambda)$, is called the co-spectrum and the imaginary part $q_{j k}(\lambda)$ is called the quadrature spectrum (or quad-spectrum). $\theta_{j k}(\lambda)$ is the phase spectrum and $f_{j k}(\lambda)$ is the amplitude spectrum.

A quantity of interest in the analysis of pairs of time series is the coherence, defined by

$$
\rho_{j k}(\lambda)=\frac{\left|f_{j k}(\lambda)\right|}{\left[f_{j j}(\lambda) f_{k k}(\lambda)\right]^{1 / 2}}
$$

$\rho_{j k}(\lambda)$ is the analogue of the correlation coefficient between two random variables and measures (in the frequency domain) the extent of linear relationship between $x_{j}(t)$ and $x_{k}(t)$. It can be proved that $0 \leq \rho_{j k}(\lambda) \leq 1$. Linear here should be understood in the sense that there is a linear filter acting on $x_{j}(t)$ and producing $x_{k}(t)$ (see discussion below).

Let us consider the case of a single series $x_{j}(t)$ for a moment. A theorem by Bochner states that there is a non-decreasing, bounded function $F_{j}(\lambda)$ such that

$$
C_{j j}(\tau)=\int_{-\pi}^{\pi} e^{i \lambda \tau} d F(\lambda), \tau \varepsilon Z
$$

This function $F_{j}(\lambda)$ is the spectral distribution function of the series $\mathrm{x}_{\mathrm{j}}(\mathrm{t})$ and determines a spectral measure $\mathrm{F}_{\mathrm{j}}(\Delta)$, which can be written

$$
F_{j}(\Delta)=F_{j}^{d}(\Delta)+F_{j}^{c}(\Delta) \text {. }
$$

$F_{j}^{d}(\Delta)$ and $F_{j}^{c}(\Delta)$ are, respectively, the discrete and the continuous part of $\mathrm{F}_{\mathrm{j}}(\Delta)$ and they are such that

$$
\begin{aligned}
& F_{j}^{d}(\Delta)=\lambda_{k} \sum_{\varepsilon} P_{j j}\left(\lambda_{k}\right), \\
& F_{j}^{c}(\Delta)=\int_{\Delta} f_{j j}(\lambda) d \lambda ;
\end{aligned}
$$


$P_{j j}\left(\lambda_{k}\right)$ is the spectral function and $f_{j j}(\lambda)$ is the spectral density function or simply the spectrm, given by (2.6) if (2.4) holds (with $j=k$ ). It follows that $C_{j j}(\tau)$ can be written

$$
C_{j j}(\tau)=\sum_{k} e^{i \lambda \lambda_{k}^{\tau}} p_{j j}\left(\lambda_{k}\right)+\int_{\pi}^{\pi} e^{i \lambda \tau_{f j}}(\lambda) d \lambda,
$$

and we obtain a mixed spectrum, formed by spectral lines plus a continuous part (Figure 1).

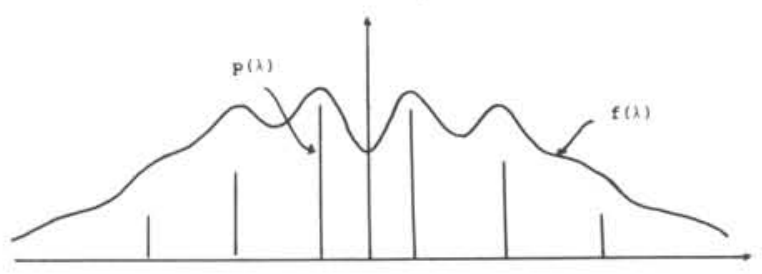

Fig. 1 - General form of the spectrum of a time series, showing the discrete part $p(\lambda)$ and the continuous part $f(\lambda)$.

A typical tidal spectrum will look like this, where the continuous part is due to the background noise and the spectral lines are due to the deterministic part of the tidal process, in general assumed to be an almost periodic function. See section 3 for a discussion on this subject.

\section{2 - THE BISPECTRUM}

Let $C_{j_{1} j_{2} j_{3}}\left(t_{1}, t_{2}, t_{3}\right)$ be the cumulant of order 3 of the p vector-valued time series $\underset{\sim}{X}=\{\underset{\sim}{X}(t), t \in Z\}$. Assume that $\underset{\sim}{X}$ is $3^{\text {rd }}$ order stationary, that is, the finite-dimensional distributions of $\underset{\sim}{\mathrm{X}}$ up to order 3 are invariant under time translations. Then it follows that

$$
C_{j_{1} j_{2} j_{3}}\left(t+\tau_{1}, t+\tau_{2}, t\right)=C_{j_{1} j_{2} j_{3}}\left(\tau_{1}, \tau_{2}\right),
$$

and if $E\left[x_{j}(t)\right]=0, j=1, \ldots, p$, then

$$
C_{j_{1} j_{2} j_{3}}\left(\tau_{1}, \tau_{2}\right)=E\left[x_{j_{1}}(t) x_{j_{2}}\left(t+\tau_{1}\right) x_{j_{3}}\left(t+\tau_{2}\right)\right]
$$


where $j_{1}, j_{2}, j_{3}=1, \ldots, p$. If $p$ is any permutation of the indices 1,2 and 3 , then it is also true that

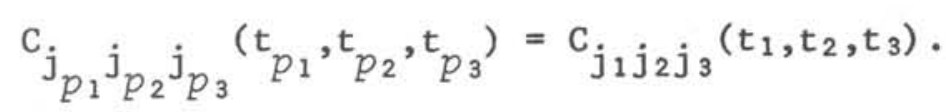

For further details on cumulants, see Brillinger, 1975, Chapter 2. If

$$
\tau_{1}, \tau_{2}\left|c_{j_{1} j_{2} j_{3}}\left(\tau_{1}, \tau_{2}\right)\right|<\infty,
$$

the bispectrum of $\underset{\sim}{\mathrm{x}}$ is defined by

$$
f_{j_{1} j_{2} j_{3}}\left(\lambda_{1}, \lambda_{2}\right)=(2 \pi)^{-2} \tau_{1}, \tau_{2} C_{j_{1} j_{2} j_{3}}\left(\tau_{1}, \tau_{2}\right) e^{-i\left(\lambda_{1} \tau_{1}+\lambda_{2} \tau_{2}\right)}
$$

for $-\infty<\lambda_{1}, \lambda_{2}<+\infty$, and this is bounded, uniformly continuous and periodic with period $2 \pi$ with respect to $\lambda_{1}$ and $\lambda_{2}$. By $(2.17)$ we have

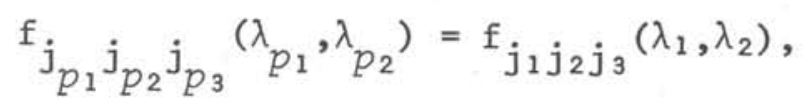

and if the components of $\underset{\sim}{\mathrm{X}}$ are real, then

$$
f_{j_{1} j_{2} j_{3}}\left(\lambda_{1}, \lambda_{2}\right)=f_{j_{1} j_{2} j_{3}}\left(-\lambda_{1},-\lambda_{2}\right) \text {. }
$$

Also, in the case of the bispectrum of a single series $x_{j}(t)$, we have

$$
f_{j j j}\left(\lambda_{1}, \lambda_{2}\right)=f_{j j j}\left(-\lambda_{1}-\lambda_{2}, \lambda_{2}\right)=f_{j j j}\left(\lambda_{1},-\lambda_{1}-\lambda_{2}\right) .
$$

If $x_{i}(t) \neq x_{j}(t) \neq x_{k}(t)$ we call $f_{i j k}\left(\lambda_{1}, \lambda_{2}\right)$ the trivariate bispectrum of $x_{i}(t), x_{j}(t)$ and $x_{k}(t)$; if $x_{i}(t)=x_{j}(t) \neq x_{k}(t), f_{i i k}\left(\lambda_{1}, \lambda_{2}\right)$ is the cross-bispectrum of $x_{i}(t)$ and $x_{k}(t)$; finally, if $x_{i}(t)=x_{j}(t)=x_{k}(t)$, 
$f_{i i i}\left(\lambda_{1}, \lambda_{2}\right)$ is the auto-bispectrum of $x_{i}(t)$. We can also write $f_{j_{1} j_{2} j_{3}}\left(\lambda_{1}, \lambda_{2}\right)$ as $\mathrm{f}_{\mathrm{j}_{1} \mathrm{j}_{2} \mathrm{j}_{3}}\left(\lambda_{1}, \lambda_{2}, \lambda_{3}\right)$, with the understanding that $\lambda_{1}+\lambda_{2}+\lambda_{3}=0$, that is, the bispectrum has support only on the manifold $\sum_{i=1}^{3} \lambda_{i}=0$ of the $\left(\lambda_{1}, \lambda_{2}, \lambda_{3}\right)$-space. If (2.18) is not satisfied we can formulate a general theory for polyspectra (Brillinger \& Rosenblatt, 1967; Gasser, 1972).

The records of ocean waves, in a given position, may be considered as realizations of Gaussian stationary processes. For these processes the spectrum is adequate to analyse their probabilistic structure. It is known that for Gaussian process the cumulant spectra of order greater than two vanish. Therefore, the bispectrum (and polyspectra of higher order) can be useful to detect deviations from a Gaussian process. The complexity of ocean waves, for example, makes necessary to consider moments greater than 2 and, in consequence, higher-order spectra. On the other hand, there have been relatively few app1ications of polyspectra, mainly due to difficulties of interpretations, time and computer work programming. Brillinger, 1965 says: "Experience with real random variables indicates that higher order moments are not efficient estimates of scientifically relevant parameters; consequently as the specifications of stochastic processes become tighter, polyspectra are likely to prove less pertinent in a similar manner."

Applications have been restricted to auto-bispectrum. Hasselmann et al., 1965, have analysed the bispectrum of wave records at a single station. Brillinger \& Rosenblatt, $1967 \alpha$, have considered $2^{\text {nd }}$ and $3^{\text {rd }}$ order spectra of the sunspot numbers series.

\section{3 - homogeneous PROCESSES ON A SPHERE}

Let $X=\left\{X(P, t): P \varepsilon S_{2}, t \in Z\right\}$ be a real-valued stochastic process defined on the unit sphere $S_{2}$ of $\mathbb{R}^{3}$. We assume that $\mathrm{X}$ is continuous in quadratic mean (q.m.), with finite moments of all orders and (weakly) stationary with respect to $t$ and homogeneous with respect to $P$, that is

(i) $\quad \mathrm{E}\{\mathrm{X}(\mathrm{P}, \mathrm{t})\}=$ constant,
(ii) $\operatorname{Cov}\{\mathrm{X}(\mathrm{P}, \mathrm{t}+\mathrm{s}), \mathrm{X}(\mathrm{Q}, \mathrm{s})\}=\mathrm{R}(\theta, \mathrm{t})$, 
where $\theta$ is the angular distance between $P$ and $Q$. We assume that the mean (2.23) is zero. It then follows that

$$
X(P, t)=\sum_{n=0}^{\infty} \sum_{j=-n}^{n} Z_{n j}(t) Y_{n j}(P),
$$

with

$$
Z_{n j}(t)=\int_{S_{2}} X(P, t) Y_{n j}(P) d P,
$$

where $d P$ is the measure on $S_{2}$ and $\left\{\mathrm{Y}_{n j},-n \leq j \leq n\right\}$ are the spherical harmonics of order $n \geq 0$. In (2.25) the series is assumed to be convergent in $\mathrm{q} . \mathrm{m}$. and the integral in $(2.26)$ is a stochastic integral in the q.m. sense. See Yaglow, 1961, for a full treatment of the above expressions. See also Roy, 1976.

The process $z_{n j}(t)$ is such that

$$
E\left\{z_{n j}(t+s) z_{m k}(s)\right\}=\delta_{n m} \delta_{j k} \int_{-\pi}^{\pi} e^{i \lambda t} d F_{n}(\lambda),
$$

and the covariance function $\mathrm{R}(\theta, \mathrm{t})$ is given by

$$
R(\theta, t)=(4 \pi)^{-1} \sum_{n=0}^{\infty}(2 n+1) P_{n}(\cos \theta) \int_{-\pi}^{\pi} e^{i \lambda t} d F_{n}(\lambda),
$$

where $P_{n}($.$) are the Legendre polynomials of degree n$ and $\left\{F_{n}(\lambda)\right\}$ is a sequence of real, bounded, non-decreasing functions. $F_{n}(\lambda)$ is the spectral distribution function of $\mathrm{x}$.

Applications of stochastic processes on a sphere have been done by Jones, 1963 and Cohen \& Jones, 1969. They have applied the methodology of this section to the problem of meteorological forecasts.

\section{4 - FILTERING, SAMPLING AND ALIASING}

In many situations it is necessary to filter the original series before proceeding to the analysis. For instance, the spectrum of a tidal record has usually significant power in a band of frequencies, hence we can use a band- 
pass filter in order to isolate the most important tidal components.

By a filter we mean a mechanism $\mathcal{F}$ which transforms a time series $x(t)$ in another time series $\mathrm{Y}(\mathrm{t})$ and we indicate this by $\mathrm{Y}(\mathrm{t})=\mathcal{F}[\mathrm{X}(\mathrm{t})]$.

$\mathcal{F}$ is a linear filter if:

(i) $f\left[\alpha_{1} X_{1}(t)+\alpha_{2} X_{2}(t)\right]=\alpha_{1} \mathcal{\exists}\left[X_{1}(t)\right]+\alpha_{2} \mathcal{F}\left[X_{2}(t)\right]$, for $\alpha_{1}, \alpha_{2}$ constant;

(ii) if $Y(t)=\mathcal{F}[X(t)]$, then $Y(t+\tau)=\mathcal{F}[X(t+\tau)]$.

Property (i) gives the linearity of $f$ and (ii) gives the so-called timeinvariance of $\mathcal{F}$. Of special importance are the filters

$$
Y(t)=\sum_{\tau=-\infty}^{\infty} h(t-\tau) X(\tau),
$$

for stationary processes $X(t)$ and

$$
Y(P)=(2 \pi)^{-1} \int_{0}^{2 \pi} \int_{0}^{\pi} h(\theta) X(Q) \operatorname{sen} \theta d \theta d \phi,
$$

for homogeneous (time-independent) processes on a sphere, $\theta$ and $\phi$ being the latitude and longitude of $Q$. The filters (2.29) and (2.30) are completely determined by their respectivetransfer functions

$$
H(\lambda)=\sum_{\tau} h(\tau) e^{-i \lambda \tau},
$$

and

$$
H(\lambda)=\int_{0}^{\pi} P_{\lambda}(\cos \theta) h(\theta) \operatorname{sen} \theta d \theta
$$

where $P_{\lambda}(\cdot)$ is the Legendre polynomial. In case (2.29), if $f_{X X}(\lambda)$ and $f_{Y Y}(\lambda)$ are the spectra of $X(t)$ and $Y(t)$, respectively, then the simple relation $f_{Y Y}(\lambda)=|H(\lambda)|^{2} \cdot f_{X X}(\lambda)$ holds.

Suppose now we have a continuous, stationary series $\{x(t),-\infty<t<+\infty\}$ and we sample it at $j \Delta t, j \varepsilon Z, \Delta t>0$. We obtain the series

$$
x_{\Delta t}(j)=x(j \Delta t), j \varepsilon Z
$$


Through this sampling procedure some harmonics of the spectral decomposition of $X(t)$ cannot be distinguished. This is the aliasing phenomenum and if we denote by $f(\lambda)$ and $f_{\Delta t}(\lambda)$ the spectrum of $x(t)$ and $x_{\Delta t}(j)$, respective$1 y$, then it can be shown that

$$
\mathrm{f}_{\Delta \mathrm{t}}(\lambda)=\sum_{\mathrm{k}=-\infty}^{\infty} \mathrm{f}\left(\lambda+\frac{2 \mathrm{k} \pi}{\Delta \mathrm{t}}\right), \frac{-\pi}{\Delta \mathrm{t}}<\lambda \leq \frac{\pi}{\Delta \mathrm{t}} .
$$

For any $\lambda$ in the interval $\left[\frac{-\pi}{\Delta t}, \frac{\pi}{\Delta t}\right]$, the frequencies $\lambda+\frac{2 k \pi}{\Delta t}, k= \pm 1, \pm 2, \ldots$ are called the aliases of $\lambda$. The frequency $\frac{\pi}{\Delta t}$ (in radians per unit of time) is called the Nyquist frequency. If frequency is given in cycles per unit of time, the Nyquist frequency is $1 / 2 \Delta t$. If $\Delta t$ is small in such a way that $f(\lambda) \simeq 0$ for $|\lambda|>\frac{\pi}{\Delta t}$ then $f_{\Delta t}(\lambda)$ and $f(\lambda)$ will be essentially the same. On the other hand, if there is no interest in $f(\lambda)$ for $|\lambda|>\frac{\pi}{\Delta t}$, then we can avoidaliasing through the applications of a low-pass filter, which attenuates or eliminates the energy at high frequencies. For details on the effect of aliasing in the case of stochastic processes on a sphere see Hannan, 1966.

The problem of aliasing also appears in connection with bispectrum. If we have two continuous series $X(t)$ and $Y(t)$, for example, and $\lambda_{N}^{(x)}=\frac{\pi}{\Delta x}$, $\lambda_{\mathrm{N}}^{(\mathrm{y})}=\frac{\pi}{\Delta \mathrm{y}}$ are the respective Nyquist frequencies, then the cross-bispectra of the continuous series and of the sample series will be essentially the same if the $\mathrm{f}_{\mathrm{YXX}}\left(\lambda_{1}, \lambda_{2}\right) \simeq 0$ for $\left|\lambda_{1}\right|>\lambda_{\mathrm{N}}^{(\mathrm{x})}$ and $\left|\lambda_{2}\right|>\lambda_{\mathrm{N}}^{(\mathrm{y})}$. Under regularity conditions, it can be shown that

$$
\mathrm{f}_{\mathrm{YXX}}^{(\Delta t)}\left(\lambda_{1}, \lambda_{2}, \lambda_{3}\right)=\sum \mathrm{f}_{\mathrm{YXX}}\left(\lambda_{1}+\frac{2 \pi j_{1}}{\Delta t}, \lambda_{2}+\frac{2 \pi j_{2}}{\Delta t}, \lambda_{3}+\frac{2 \pi j_{3}}{\Delta t}\right),
$$

3

for $\sum_{j=1} \lambda_{j}=0(\bmod 2 \pi)$, the sum extended over all $j_{\alpha}$ such that

$$
\sum_{\alpha=1}^{3}\left(\lambda_{2}+\frac{2 \pi j_{\alpha}}{\Delta t}\right)=0 \text { and } \Delta x=\Delta y=\Delta t \text {. }
$$

For practical aspects of computations of the bi-spectra see Brillinger \& Rosenblatt, 1967a and Gasser, 1972. To give an idea, for one series X(t), the bi-spectrum $\mathrm{f}_{\mathrm{Xxx}}\left(\lambda_{1}, \lambda_{2}\right)$ is computed in the triangular area of vertices $(0,0,0),(\pi, 0,-\pi)$ and $\left(\frac{\pi}{2}, \frac{\pi}{2},-\pi\right)$, in the case $\lambda_{1}+\lambda_{2}+\lambda_{3}=0$ 


\section{5 - ESTIMATION PROCEDURES}

In this section we restrict our attention to the problem of estimating spectra and bispectra. There are basically two approaches for estimating spectral parameters. The first uses estimates which are obtained through a smoothing of the sample covariance function. The second uses estimates which are weighted averages of periodogram ordinates.

Suppose we have observations $\underset{\sim}{X}(t), t=1, \ldots, N$ of the $p$ vector-valued process (2.3). Define the vector $(\mathrm{pxl})$ of finite Fourier transforms

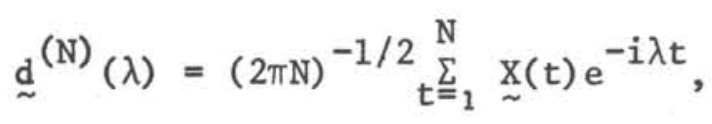

for $-\infty<\lambda<+\infty$. Usually this is computed for frequencies $\lambda_{\nu}=\frac{2 \pi \nu}{\mathrm{N}}$, for $-\left[\frac{N-1}{2}\right] \leq \nu \leq\left[\frac{N}{2}\right]$. Let $\mathrm{E}[\underset{\sim}{\mathrm{X}}(\mathrm{t})]=\underset{\sim}{0}$ and $\underset{\sim}{\mathrm{f}}(\lambda)$ be the matrix of spectra $f_{j k}(\lambda), j, k=1, \ldots, p$. Under regularity conditions (Brillinger, 1975) the random variable $\underset{\sim}{d}{ }^{(N)}(\lambda)$ has an asymptotic distribution which is a multivariate complex normal distribution $\mathrm{N}_{\mathrm{p}}^{\mathrm{c}}(\underset{\sim}{0} \underset{\sim}{\mathrm{f}}(\lambda))$, if $\lambda \neq 0, \pi$. If $\lambda=0$ or $\lambda=\pi$, the asymptotic distribution is a $N_{p}(\underset{\sim}{0} \underset{\sim}{f}(\lambda))$.

Let $d_{j}^{(N)}(\lambda)$ be the $j-$ th component of $\underset{\sim}{d}(N)(\lambda)$ and define the crossperiodogram of the series $x_{j}(t)$ with the series $x_{k}(t)$ by

$$
I_{j k}^{(N)}(\lambda)=d_{j}^{(N)}(\lambda) \cdot \overline{d_{k}^{(N)}(\lambda)} \text {. }
$$

If $\mathrm{j}=\mathrm{k}$ we have the periodogram of $\mathrm{x}_{\mathrm{j}}(t)$ given by

$$
I_{j j}^{(N)}(\lambda)=\left|d_{j}^{(N)}(\lambda)\right|^{2}=(2 \pi N)^{-1}\left|\sum_{t=1}^{N} x_{j}(t) e^{-i \lambda t}\right|^{2} \text {. }
$$

It can be proved that $I_{j j}^{(N)}(\lambda)$ is an asymptotically unbiased estimator of $f_{j j}(\lambda)$, but it is not consistent, since its variance is (asymptotically) equal to $f_{j j}^{2}(\lambda)$, for $\lambda \neq 0, \pi$ and $2 f_{j j}^{2}(\lambda)$, for $\lambda=0$ or $\lambda=\pi$. Moreover, $I_{j j}^{(N)}(\lambda)$ has an asymptotic distribution proportional to a chi-square variable with 2 degrees of freedom, if $\lambda \neq 0, \pi$ and proportional to a chi-square variable with 1 degree of freedom if $\lambda=0$ or $\lambda=\pi$. 
Let $\widehat{C}_{j k}(\tau)$ be an estimate of the cross-covariance function $C_{j k}(\tau)$ defined by

$$
\vec{C}_{j k}(\tau)=\left\{\begin{array}{l}
N^{-1}{\underset{t=1}{N} \bar{\Sigma}_{j}^{\tau}}_{\widehat{C}_{k j}}(t+\tau), \quad \tau=-1,-2, \ldots,-N+1 \\
0, \quad|\tau| \geq N .
\end{array}\right.
$$

Let $w_{M}(\tau)$ be a sequence of weights such that:

$$
\begin{aligned}
& \text { (i) } 0 \leq w_{M}(\tau) \leq w_{M}(0)=1 ; \\
& \text { (ii) } w_{M}(-\tau)=w_{M}(\tau) \text {, for all } \tau, \\
& \text { (iii) } w_{M}(\tau)=0 \text {, for }|\tau|>M .
\end{aligned}
$$

The function $\mathrm{w}_{\mathrm{M}}(\tau)$ is called a $\mathrm{lag}$ window and its Fourier transform

$$
W_{M}(\lambda)=(2 \pi)^{-1} \sum_{\tau=-\infty}^{\infty} W_{M}(\tau) e^{-i \lambda \tau}
$$

is called a spectral window and has the properties:

$$
\begin{aligned}
& \text { (i) } \mathrm{W}_{M}(-\lambda)=\mathrm{W}_{M}(\lambda) \text {; } \\
& \text { (ii) } \int_{-\pi}^{\pi_{M}} \mathrm{~W}_{M}(\lambda) \mathrm{d} \lambda=1 .
\end{aligned}
$$

There are many windows used in practice, such as the Bartlett, Tukey, Parzen windows, etc. See Jenkins \& Watts, 1968 for details.

If we collect the $\widehat{C}_{j k}(\tau)$ in the pxp matrix $\underset{\sim}{\widehat{C}}(k)$, we define the smoothed covariance estimate of $\underset{\sim}{f}(\lambda)$ by

$$
\underset{\sim 1}{\vec{f}_{1}}(\lambda)=(2 \pi)^{-1} \sum_{\tau=-\infty}^{\infty} e^{-i \lambda \tau} \cdot w_{M}(\tau) \underset{\sim}{\overparen{C}}(\tau)
$$


It can be seen, from the convolution properties of the Fourier transform and using a Riemann approximating sum for an integral (Koopmans, 1974, p. 267 ), that $\underset{\sim}{f_{1}}(\lambda)$ can be written in the asymptotically (in distribution sense) equivalent form

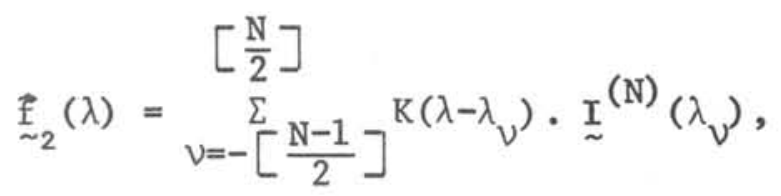

where $\lambda_{\nu}=\frac{2 \pi \nu}{N}$ and $K(\lambda)$ is a symmetric, periodic, real-valued weight function for which $\sum K\left(\lambda_{V}\right)=1$. Here $\underset{\sim}{I}{ }^{(N)}(\lambda)=\left[I_{j k}^{(N)}(\lambda)\right]$ is the matrix of crossperiodograms (2.36). Estimators given by (2.43) are called smoothed periodogram estimators. The estimators $(2.42)$ and $(2.43)$ are asymptotically unbiased and, under regularity conditions on $M, N$ and $K(\lambda)$, they have, asymptotically, a distribution which is a constant multiple of a chi-square variable with $\mathrm{r}$ degrees of freedom. The parameter $r$ is called the equivalent degrees of freedom of the estimator and depends on $K(\lambda)$, since $K(\lambda) \simeq \frac{2 \pi_{N}}{N} W_{M}(\lambda)$. If we use (2.42), then $r=N / \int_{-\pi} \pi_{M}^{2}(\lambda) d \lambda$ and if we use (2.43), $r=2 / \Sigma K^{2}\left(\lambda_{\nu}\right)$.

As estimates of the coherence $\rho_{j k}(\lambda)$ and of the phase $\theta_{j k}(\lambda)$ we take

$$
\hat{\rho}_{j k}(\lambda)=\frac{\left[\hat{f}_{j k}(\lambda)\right]}{\left[\hat{f}_{j j}(\lambda) f_{k k}(\lambda)\right]^{1 / 2}}
$$

and

$$
\theta_{j k}(\lambda)=\operatorname{tg}^{-1}\left[\frac{\hat{q}_{j k}(\lambda)}{\hat{c}_{j k}(\lambda)}\right],
$$

where $\hat{c}_{j k}(\lambda)=\operatorname{Re} \hat{f}_{j k}(\lambda), \widehat{q}_{j k}(\lambda)=\operatorname{Im} \hat{f}_{j k}(\lambda)$ and $\hat{f}_{j k}(\lambda)$ is given by (2.42) or $(2.43)$.

Approximate confidence intervals for the spectrum, coherence and phase can be obtained using the asymptotic distributions of the spectral estimates. It is convenient to consider a confidence interval for the log-spectral density, since this interval will have a constant width for all frequencies. 
It is given by

$$
\log \frac{r}{b}+\log \hat{f}(\lambda) \leq \log f(\lambda) \leq \log \frac{r}{a}+\log \hat{f}(\lambda),
$$

where $a$ and $b$ are obtained from the tables of $a \chi^{2}(r)$ by

$$
P\left(X^{2}(r) \leq a\right)=\frac{1-\gamma}{2}, P\left(X^{2}(r) \leq b\right)=\frac{1+\gamma}{2}
$$

$\gamma$ being the confidence coefficient. The constant width of the interval is $\log \frac{b}{a}$. See Koopmans, 1974, for the expressions for the confidence intervals for the coherence and phase.

Turning to the bispectrum, define the third-order periodogram by

$$
I_{j_{1} j_{2} j_{3}}^{(N)}\left(\lambda_{1}, \lambda_{2}, \lambda_{3}\right)=(2 \pi)^{-2} N^{-1} \cdot d_{j_{1}}^{(N)}\left(\lambda_{1}\right) d_{j_{2}}^{(N)}\left(\lambda_{2}\right) d_{j_{3}}^{(N)}\left(\lambda_{3}\right) \text {, }
$$

where $\lambda_{1}+\lambda_{2}+\lambda_{3}=0$. As an estimate of the bispectrum $f_{j_{1} j_{2} j_{3}}\left(\lambda_{1}, \lambda_{2}, \lambda_{3}\right)$ we take the weighted periodogram

$$
\mathrm{f}_{\mathrm{j}_{1} \mathrm{j}_{2} \mathrm{j}_{3}}^{(\mathrm{N})}\left(\lambda_{1}, \lambda_{2}, \lambda_{3}\right)=\iint_{-\infty}^{+\infty} \int \mathrm{W}_{M}\left(\lambda_{1}-\alpha_{1}, \lambda_{2}-u_{2}, \lambda_{3}-\alpha_{3}\right) I_{j_{1} j_{2} j_{3}}^{(N)}\left(\alpha_{1}, \alpha_{2}, \alpha_{3}\right) \delta\left(\alpha_{1}+\alpha_{2}+\alpha_{3}\right) d \alpha_{1} d \alpha_{2} d \alpha_{3},
$$

where $\mathrm{W}_{\mathrm{N}}($.$) is a continuous weighting function and \delta($.$) is the Dirac function.$ For computations, (2.48) can be written in a form similar to (2.43). For details, see Brillinger \& Rosenblatt, 1967. The estimate (2.48) is asymptotically unbiased off minor submanifolds and asymptotically normally distributed as $\mathrm{N} \rightarrow \infty$.

A further aspect that deserves attention in the estimation of spectra and bi-spectra is the problem of complex-demodulation. Let $x(t)$ be a realvalued series and consider

$$
x_{0}(t)=L\left[X(t) e^{i \lambda_{0} t}\right]
$$

$\mathrm{Xo}(\mathrm{t})$ is called the complex-demodulate series centered at frequency $\lambda o$. Here $\mathrm{L}$ is a low-pass filter which can be chosen in a variety of forms. If $\Delta \lambda$ is 
the bandwidth of $\mathrm{L}$, then an estimate of the spectrum of $X(t)$ in $\lambda_{0} \pm \Delta \lambda$ is given by $T^{-1} \cdot \int_{t_{1}}^{t_{2}}\left|x_{0}(t)\right|^{2} d t$, where $T$ is the lenght of a record of $X(t)$ from $t=t_{1}$ up to $t=t_{2}$. As an estimate of the (trivariate) bi-spectrum of $x_{1}(t)$, $x_{2}(t)$ and $X_{3}(t)$ we take

$$
\hat{f}_{123}\left(\lambda_{1}, \lambda_{2}, \lambda_{3}\right)=N^{-1} \sum_{t=1}^{N} x_{1, \lambda_{1}}(t) x_{2, \lambda_{2}}(t) x_{3, \lambda_{3}}(t),
$$

where $\lambda_{1}+\lambda_{2}+\lambda_{3}=0, \quad N$ is the number of available values of the complex demodulates and $x_{i, \lambda i}(t)$ is the complex demodulate of $x_{i}(t)$ at frequency $\lambda_{i}, i=1,2,3$. An advantage of this procedure is that we can obtain the estimated cross-spectrum at the same time:

$$
\hat{f}_{12}\left(\lambda_{1}, \lambda_{2}\right)=N^{-1} \sum_{t=1}^{N} x_{1, \lambda_{1}}(t) x_{2, \lambda_{2}}(t),
$$

$\lambda_{1}+\lambda_{2}=0$. For details, see Godfrey, 1965 and Tukey, 1961 .

\section{THEORETICAL AND REAL TIDES}

Newton introduced the concept of the equilibrium tide (the tide that would have been produced if the rigid and spherical earth were covered entirely by the oceans) and this has been used as the basis for later developments in tidal theory by Laplace, Darwin, Doodson (1921) and Cartwright \& Edden, 1973.

In real tides, as measured by tide gauges at a fixed point on earth, the equilibrium is never attained and the record tidal wave is a superposition of effects such as the revolution of the "fixed point" through the deformed water surface (by the tidal tractive forces of the moon and the sun) from the spherical shape, the modification of the deformed water surface determined astronomically by the moon and sun positioning relative to the earth, shallow water effects, radiation effects, atmospheric influences and others.

Earth has an angular velocity which is far greater than the rate of modification of the deformed water level. This time rate is directly related 
to the orbits of the moon and the sun (frame of reference put on earth) and in the Equilibrium Theory of Tides it is described by the tide generating potential (Munk \& Cartwright, 1966) $V(P, t)=V(\theta, \phi, t)$ given by

$$
\mathrm{V}(\theta, \phi, t)=\sum_{n=2}^{\infty}{ }_{m=-n}^{n} Y_{n}^{m}(\theta, \phi) \cdot c_{n}^{m}(t),
$$

which can further be written in the form

$$
\begin{aligned}
\mathrm{V}(\theta, \phi, t) & =a \frac{M_{L}}{M_{E}} \sum_{n=2}^{\infty} \sum_{m}^{n} \frac{4 \pi}{2 n+1}\left(\frac{a}{R_{L}}\right)^{n} Y_{n}^{m}(\theta, \phi) Y_{n}^{m}\left(\theta_{L}, \phi_{L}\right) *+ \\
& +a \frac{M_{S}}{M_{E}} \sum_{n=2}^{\infty} \sum_{m}^{\infty} \frac{n}{2 n+1}\left(\frac{a}{R_{S}}\right)^{n} Y_{n}^{m}(\theta, \phi) Y_{n}^{m}\left(\theta_{S}, \phi_{S}\right) *
\end{aligned}
$$

In equation (3.2), $\theta$ and $\phi$ are the colatitude and longitude of the point $P$ on the earth's surface; $t$ is the time, $a$ is the radius of the earth and $M_{E}$ is its mass; $\theta_{L}$ and $\phi_{L}$ are the colatitude and longitude of the moon, $R_{L}$ its distance from the centre of the earth and $M_{L}$ its mass. Similar terms with the subscript $\mathrm{S}$ refer to the sun. The terms $\mathrm{Y}_{\mathrm{n}}^{\mathrm{m}}$ are the spherical harmonics, defined by

$$
\mathrm{Y}_{\mathrm{n}}^{\mathrm{m}}(\theta, \phi)=\left(\frac{2 \mathrm{n}+1}{4 \pi}\right)^{1 / 2}\left[\frac{(\mathrm{n}-|\mathrm{m}|) !}{(\mathrm{n}+|\mathrm{m}|) !}\right]^{1 / 2} \mathrm{P}_{\mathrm{n}}^{|\mathrm{m}|}(\cos \theta) \mathrm{e}^{\mathrm{im} \phi}
$$

$\mathrm{P}_{\mathrm{n}}^{\mathrm{m}}$ being the associated Legendre functions

$$
P_{n}^{m}(x)=\frac{\left(1-x^{2}\right)^{m / 2}}{2^{n} \cdot n !} \cdot \frac{d^{m+n}}{d x^{m+n}}\left(x^{2}-1\right)^{n} .
$$

Values of $\left(\theta_{L}, \phi_{L}\right)$ and $\left(\theta_{S}, \phi_{S}\right)$ give the positioning of the moon and the sun relative to the earth uniquely. Terms for $n=0$ and $n=1$ in (3.1) are neglected as they do not contribute to the tide generating potential at $P$. 
Periodical parameters of the orbits of the moon and the sun used for the time description of $\left(\theta_{L}, \phi_{L}\right)$ and $\left(\theta_{S}, \phi_{S}\right)$ are:

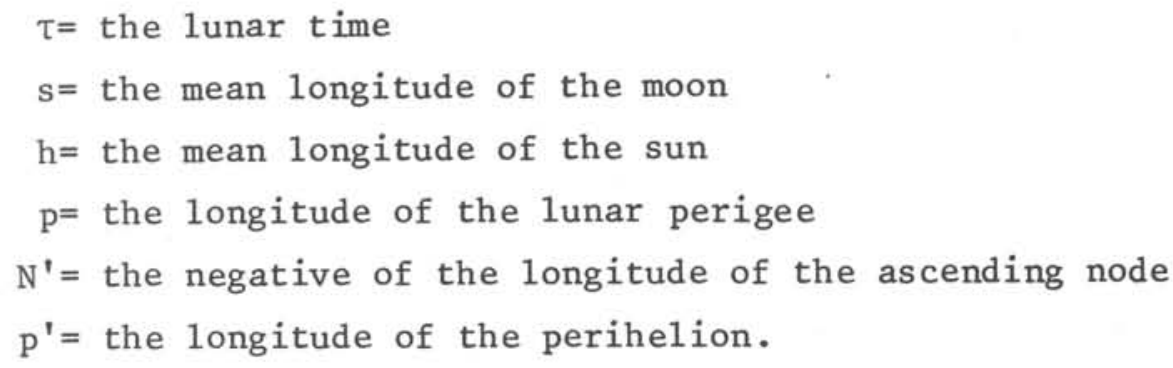

Here, $\mathrm{p}^{\prime}$ completes one revolution in 20,900 years, $\mathrm{N}^{\prime}$ in 18.61 years, $\mathrm{p}$ in 8.85 years, $h$ in one year, $s$ in one lunar month. If we write (3.1) as $V(P, t)=V\left(\theta_{L}, \phi_{L}, t\right)+V\left(\theta_{S}, \phi_{S}, t\right)$, then the resulting expansion of the two terms in the right hand-side of this equality in terms of the variables (3.5) are given as a sum of cosines and sines of

$$
i \tau+j s+k h+1 p+m N^{\prime}+n p^{\prime},
$$

where the weights $\{i, j, k, 1, m, n\}$ are the Doodson's numbers and they varie from -6 to +6 individually (Godin, 1972). A combination of integers $\{i, j, k, 1, m, n\}$ characterizes a constituent with frequency

$$
\lambda=\mathrm{i} \dot{\tau}+\mathrm{j} \dot{\mathrm{s}}+\mathrm{k} \dot{\mathrm{h}}+1 \dot{\mathrm{p}}+\mathrm{m} \dot{\mathrm{N}}+\mathbf{n} \dot{\mathrm{p}}
$$

of the tidal spectrum and in the Harmonic Method of tidal analysis the search for its phase and amplitude is the prior objective.

As already mentioned, modifications of the shape of the disturbed sea level, due to the action of the generating tidal potential described by (3.1) are much slower than the rate with which the observing point $P=(\theta, \phi)$ is displaced, so P "moves" through the disturbed sea level.

The tidal record of a time lenght of one day contains (for the great 
majority of ports) the recording of a predominant semi-diurnal tide which is representative of a day sample of the "shape" of the tide generating potential. Its spectrum would have a predominant line ( $\mathrm{M}_{2}$ group of constituents) at $\lambda=2 \dot{\tau}+0 \dot{s}+0 \dot{h}+0 \dot{p}+0 \dot{N}^{\prime}+0 \dot{p}^{\prime} \cong 0.08$ cycles/hour, plus other minor contributors. A set of dayly samples covering the period of one lunar month ( $\mathrm{s}$ is associated with this period of approximately 28 days) would have in each day record a predominant semidiurnal variation, but with modulated amplitudes from one day to another, due to the variations of the "shape" of the water level (shape of the tide potential at $\mathrm{P}=(\theta, \phi)$ ), as the moon has completed during this period one orbit lenght and the earth about $1 / 12$ of its path around the sun. Increasing the number of samples other features of the tide generating potential are added to the semi-diurnal wave carrier and they can be analysed by Fourier methods .

Usually it is assumed that the spectrum of $\mathrm{V}(\mathrm{t})$ is a line spectrum. Munk \& Cartwright, 1966, noticed however that the spectrum of tidal records consists of peaks energing from a continuous spectrum. The continuous part is due to background noise, particularly in low frequencies.

Hence, a reasonable model is the following. We assume that a tidal record is a superposition of a strong deterministic process plus a weak random process. Specifically, let $\varepsilon(t)$ a stationary, ergodic process, with mean zero and covariance function $C_{\varepsilon \varepsilon}(\tau)$. Let $x(t)$ be a non-random, real function of $t$, for which the Wiener covariance function

$$
C_{x x}(\tau)=\lim _{T \rightarrow \infty} \frac{1}{2 T} \int_{-T}^{T} x(t) x(t+\tau) d t
$$

exists and it is finite for every $\tau$. Assume also that

$$
\lim _{t \rightarrow \infty} \frac{1}{2 T} \int_{-T}^{T} x(t+\tau) \varepsilon(t) d t=0
$$

where the limits are in $q . m$. sense. If $Z(t)$ is the tidal height, then write

$$
Z(t)=x(t)+\varepsilon(t)
$$


$-\infty<t<+\infty$. It follows that

$$
\mathrm{C}_{\mathrm{zZ}}(\tau)=\mathrm{C}_{\mathrm{Xx}}(\tau)+\mathrm{C}_{\varepsilon \varepsilon}(\tau)
$$

and $\mathrm{Z}(\mathrm{t})$ will have a spectral distribution function (in Wiener's sense)

$$
F_{z}(\Delta)=F_{x}(\Delta)+F_{\varepsilon}(\Delta) \text {. }
$$

Usually, $\mathbf{x}(t)$ is assumed to be an almost periodic function of the form

$$
x(t)=\sum_{=-n}^{n} C e^{i \lambda} j^{t},
$$

and $\varepsilon(t)$ is assumed Gaussian, in such a way that the spectrum of $\varepsilon(t)$ is continuous and the spectrum of $\mathrm{x}(\mathrm{t})$ is discrete.

In the notation of section 2.1 ,

$$
p_{Z Z}(\lambda)=p_{x x}(w), f_{Z Z}(\lambda)=f_{\varepsilon \varepsilon}(\lambda),
$$

that is, the spectrum of $\mathrm{Z}(\mathrm{t})$ has the form of Figure 1 .

The theoretical and real line spectra for the port of Cananeia (Lat. $25^{\circ} 2^{\prime} \mathrm{S}$, 1ong. $48^{\circ} \mathrm{W}$ ) are shown in Figure 2. Comparison of them shows that real tides are ordinarily different in amplitude (and also in phase).

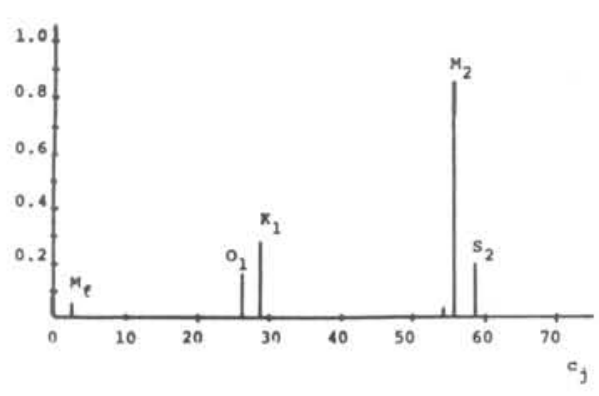

Fig. $2 b$ - Line spectrum for Cananëia, 29 days of hourly observations (129 April, 1975). The $\mathrm{c}_{j}$ 's are such that $c_{j}=\lambda_{j} \cdot T$, where $T$ is the period of observations and $\lambda_{j}$ are given by $(3.6)$.
Fig. $2 a$ - Normalized theoretical li ne spectrum for Cananéia $\left(25^{\circ} 01^{\prime} \mathrm{S}, 47^{\circ} 55^{\prime} \mathrm{W}\right)$.

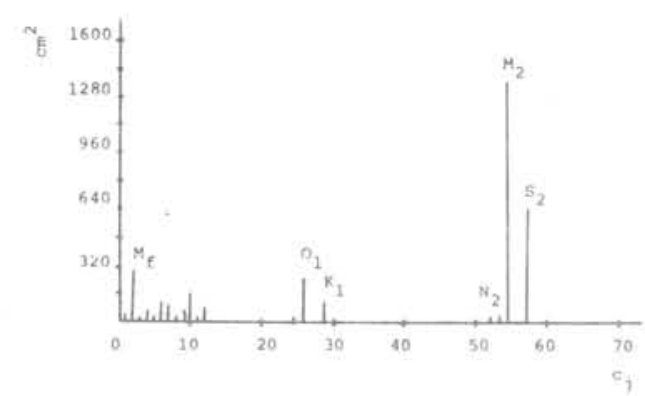


For the theoretical spectrum we followed Godin, 1972. The real line spectrum is a single use of (2.35) on a 29 days record lenght. Sampling intervals of the tidal records were so to give a 0.5 cy/hour Nyquist frequency but for Figure 2 only 75 Fourier components were taken. In Figure 3 we have

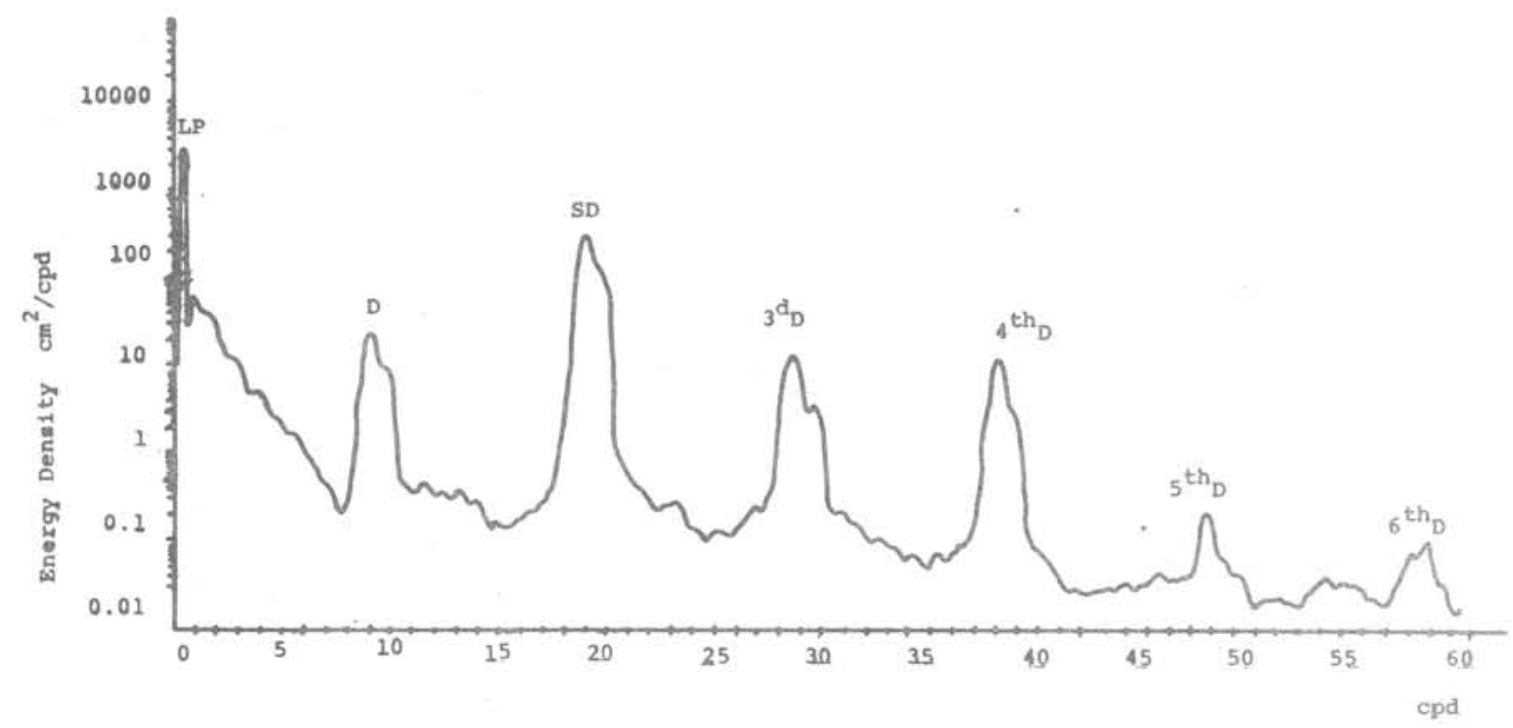

Fig. 3 - Estimated spectrum for Cananeia, with one year of hourly observations.

the spectrum of $\mathrm{Z}(\mathrm{t})$ given by (3.9), obtained from a record lenght of one year, using a smoothed procedure and a FFT algorithm (Franco \& Rock, 1971), with 8,192 digitized points .

The higher order peaks obtained are not directly due to the tidal potential but to the distortions of the tidal wave as it looses energy at the shelf areas. Open ocean records are freer of these effects. The higher order frequencies ( $3^{\text {rd }}, 4^{\text {th }}$, etc, diurnal frequencies) are the results of the important tidal constituents $\mathrm{M}_{1}, \mathrm{~K}_{1}, \mathrm{M}_{2}, \mathrm{~S}_{2}$, etc (Doodson, 1957) and they can be expressed as sums and differences of these.

The record lenght is fundamental for separating the tidal constituents predicted by (3.1) from the semidiurnal wave carrier. Two constituents are considered resolved if

$$
\left|\lambda_{1}-\lambda_{i+1}\right| \geq \frac{1}{N \cdot \Delta t}
$$


where $\mathrm{N}$ is the number of digitized values of the record and $\Delta t$ is the sampling interval.

Some of the longest records (approximately 200 years) are reported in Cartwright, 1971, but even these are insufficient to analyse for $\dot{p}^{\prime}$. Tidal analysis of short records (7 days) for some constituents is given in Franco, 1964 .

The aliasing phenomenum is not often seen in tidal analysis as $\Delta t=1$ hour (standart sampling interval normally adapted for records already instrumentally filtered for waves, swells and other higher frequencies effects) is sufficiently small for most of the records. However when shallow water components are small but necessary for prediction purposes (as seen in Figure 3, a great portion of the tidal energy is at frequencies higher than those of the group of semi-diurnals) aliasing can be used to one's advantage as in Horn, 1960 .

Estimates of the spectrafor every port may be different for many reasons, but in all cases they are based upon the same frequencies $\lambda$ determined by (3.6). The phases of the constituents may all be related, for every port, to one particular instant of time (and meridian) from when the time of $\mathrm{s}, \mathrm{h}, \mathrm{p}, \mathrm{N}^{\prime}$ and $\mathrm{p}^{\prime}$ are related (Meus, 1962). With both phases and frequencies deterministically given by astronomy, the estimates of the (real) tidal spectrum are mainly concerned with the amplitudes of the tidal constituents and the determination of real phases as they are also of practical importance.

The determination for the spectral bands are influenced by the unavoidable presence of noise and its contribution to the spectra often interferes in the separation of the spectral lines (Munk \& Hasselmann, 1964).

Tidal records are also the registrar of other non tidal effects, such as atmospheric pressure and solar radiation. Analysis of tides giving a broad look to the matter is found in Munk \& Cartwright, 1966. In this approach, records are assumed to be given by

$$
Z(t)=\int_{-\infty}^{\infty} w(\tau) V(t-\tau) d \tau+L_{-\infty}^{\infty} \mathcal{L}_{\infty}^{\infty} w\left(\tau, \tau^{\prime}\right) V(t-\tau) V\left(t-\tau^{\prime}\right) d \tau d \tau^{\prime}+\ldots
$$


where $w(\tau), w\left(\tau, \tau^{\prime}\right), \ldots$ are the impulse functions of the locality. This new approach motivated recent analises by Cartwright \& Tayler, 1971 and Cartwright \& Edden, 1973, leading to more accurate values of the theoretical tidal constituents. Formula (3.15) can be written as

$$
z(t)=\sum_{j=1}^{\infty} \int_{t_{1}} \ldots \int_{t_{j}} w_{j}\left(t_{1}, \ldots, t_{j}\right) v\left(t-t_{1}\right) \ldots v\left(t-t_{j}\right) d t_{1} \ldots d t_{j},
$$

where $w_{j}\left(t_{1}, \ldots, t_{j}\right), j=1,2, \ldots$ are assumed to be symmetric in its arguments. The Fourier transform of $w_{j}\left(t_{1}, \ldots, t_{j}\right)$ is

$w_{j}\left(\lambda_{1}, \ldots, \lambda_{j}\right)=\int_{-\infty}^{\infty} \ldots \int_{-\infty}^{\infty} w_{j}\left(t_{1}, \ldots, t_{j}\right) e^{-i\left(\lambda_{1} t_{1}+\ldots+\lambda_{j} t_{j}\right)} d t_{1} \ldots d t_{j}$,

called the $j$-th transfer function or admittances. The problem that arises is the estimation of these functions. We refer to the expansion (3.16) as a Volterra functional expansion (cf. Brillinger, 1970; 1975, and Gasser, 1972). Usually a few terms of (3.16) will be sufficient. Munk \& Cartwright, 1966, consider the linear term for tidal analysis of Honolulu and the linear and quadratic terms for the port of Newlyn. Consider, therefore,

$$
z(t)=\sum_{j=1}^{N} \int_{t_{1}} \ldots \int_{t_{j}} w_{j}\left(t_{1}, \ldots, t_{j}\right) v\left(t-t_{1}\right) \ldots v\left(t-t_{j}\right) d t_{1} \ldots d t{ }_{j}
$$

or the discrete analogue, with the integrals replaced by sums .

Under certain regularity conditions on $V(t)$ and assuming that $w_{j}\left(t_{1}, \ldots t_{j}\right) \varepsilon$ $L_{1}\left(\mathbb{R}^{j}, \mathbb{B}^{j}, \mu^{j}\right)$, it follows that the Volterra expansion (3.16) exists with probability one and $w_{j}\left(\lambda_{1}, \ldots, \lambda_{j}\right) \in L_{1}$. Here, $\mathbb{B}^{j}$ is the Borel field on $\mathbb{R}^{j}$ and $\mu^{j}$ is the Lebesgue measure in $\mathbb{R}^{j}$. Moreover, if $V(t)$ has the spectral representation

$$
V(t)=\int_{-\infty}^{\infty} e^{i \lambda t} d U(\lambda),
$$


then the spectral representation of $Z(t)$ is given by

$$
Z(t)=\sum_{j=1}^{N} \int_{\lambda} e^{i \lambda t} \int_{\lambda_{1}+\ldots \lambda_{j}=\lambda} W_{j}\left(\lambda_{1}, \ldots, \lambda_{j}\right) d U\left(\lambda_{1}\right) \ldots d U\left(\lambda_{j}\right) .
$$

For practical purposes the weights $w_{j}$ are assumed to be different from zero only for integral values of $t$, in such a way that (3.18) can be written as a sum.

The cumulant spectrum of order $k$ of $z(t)$ can be computed (see Theorem 2.10.1 of Brillinger, 1975) and in particular the cross-bispectrum between $Z(t)$ and $V(t)$, which is used for estimating $W_{2}\left(\lambda_{1}, \lambda_{2}\right)$.

We note that $V(t)$ does not have to be the tidal potential; other input functions are used, such as pressure, solar radiation, etc. For details, see Godin, 1972 and Munk \& Cartwright, 1966.

For the identification of the admittances $W_{j}$ we further assume that the drived inputs are Gaussian. This is useful because (Gasser, 1972):

(i) structures beyond the spectrum are introduced;

(ii) Gauss inputs are sufficient for time-invariant system identification;

(iii) Gaussian processes can be easily generated by computers .

It follows that $W_{1}\left(\lambda_{1}\right)$ and $W_{2}\left(\lambda_{1}, \lambda_{2}\right)$ can be estimated by

$$
\hat{\mathrm{W}}_{1}\left(\lambda_{1}\right)=\frac{\hat{\mathrm{f}}_{\mathrm{ZV}}\left(\lambda_{1}\right)}{\mathrm{f}_{\mathrm{VV}}\left(\lambda_{1}\right)}
$$

and

$$
\hat{W}_{2}\left(\lambda_{1}, \lambda_{2}\right)=\frac{\hat{\mathrm{f}}_{\mathrm{VVZ}}\left(\lambda_{1}, \lambda_{2}\right)}{2 \hat{\mathrm{f}}_{\mathrm{VV}}\left(\lambda_{1}\right) \hat{\mathrm{f}}_{\mathrm{vV}}\left(\lambda_{2}\right)}
$$


respectively, where $\hat{\mathrm{f}}_{\mathrm{ZV}}\left(\lambda_{1}\right)$ is the estimated cross-spectrum between $\mathrm{Z}(\mathrm{t})$ and $\mathrm{V}(\mathrm{t})$ and $\hat{\mathrm{t}}_{\mathrm{VVZ}}\left(\lambda_{1}, \lambda_{2}\right)$ is the estimated cross-bispectrum between $\mathrm{Z}(\mathrm{t})$ and $\mathrm{V}(\mathrm{t})$; $\hat{\mathrm{f}}_{\mathrm{VV}}\left(\lambda_{1}\right)$ is the estimated (auto)-spectrum of $\mathrm{V}(\mathrm{t})$. For the estimation of $\mathrm{W}_{\mathrm{j}}$ in general see Brillinger, 1970 and Gasser, 1972.

A measure of correlation between $\mathrm{Z}(\mathrm{t})$ and $\mathrm{V}(\mathrm{t})$ is given by the estimated coherence

$$
\hat{\rho}(\lambda)=\frac{\left|\overrightarrow{\mathrm{f}}_{\mathrm{ZV}}(\lambda)\right|}{\left[\hat{\mathrm{f}}_{\mathrm{ZZ}}(\lambda) \overrightarrow{\mathrm{f}}_{\mathrm{VV}}(\lambda)\right]^{1 / 2}}
$$

After expanding $\mathrm{V}(\mathrm{t})$ in Greenwich coordinates and substituting in (3.16) we obtain the practical scheme of the Response Method, given by

$$
\mathrm{Z}(\mathrm{t})=\sum_{k} \sum_{n} w_{n k} c_{n}(t-k \Delta \tau)+\sum_{k k^{\prime}} \sum_{n n^{\prime}} w_{n n^{\prime} k k^{\prime}} c_{n}(t-k \Delta \tau) \cdot c_{n^{\prime}}\left(t-k^{\prime} \Delta \tau\right)+\ldots
$$

Besides the estimation procedure suggested above for the $w_{j}$, we can estimate the $w_{n k}$, etc by least squares and then estimate the $w_{j}$ by Fourier transforming. In (3.24) the constants $c_{n}$ are determined by the position of the moon or of the sun. The fitting of (3.24) to the actual observations is evaluated through the prediction variance, which for the linear term is given by

$$
\operatorname{Var}\left[\mathrm{z}(\mathrm{t})-{ }_{\mathrm{k}, \mathrm{n}} \mathrm{w}_{\mathrm{nk}}{ }^{\mathrm{c}} \mathrm{n}(\mathrm{t}-\mathrm{k} \Delta \tau)\right] \text {. }
$$

For details see Munk \& Cartwright, 1966.

\section{SEASONAL VARIATIONS}

As the tides, seasonal variations of the oceans are also astronomically determined. The annually varying heating effect due to the fact that the ecliptic and the equatorial plan are at an angle of $23^{\circ}$, causes the ocean currents to become weak or strong and to change their position relative to the bottom or the continental boarders, changing in consequence other oceanic properties. 
For seasonal studies long time series are normally required but as in tidal studies the information is basically analysed for a known frequency (corresponding to the period of one year) and its theoretical spectrum would consist of a single line. Obviously real spectrum is contaminated by other features not directly seasonal but often consequences of it.

Figure 4 shows the 12 monthly mean values of temperature taken at $\left(24^{\circ} \mathrm{S}\right.$, $042^{\circ} \mathrm{W}$ ) for $0 \mathrm{~m}$ and $55 \mathrm{~m}$ of depth in coastal waters.

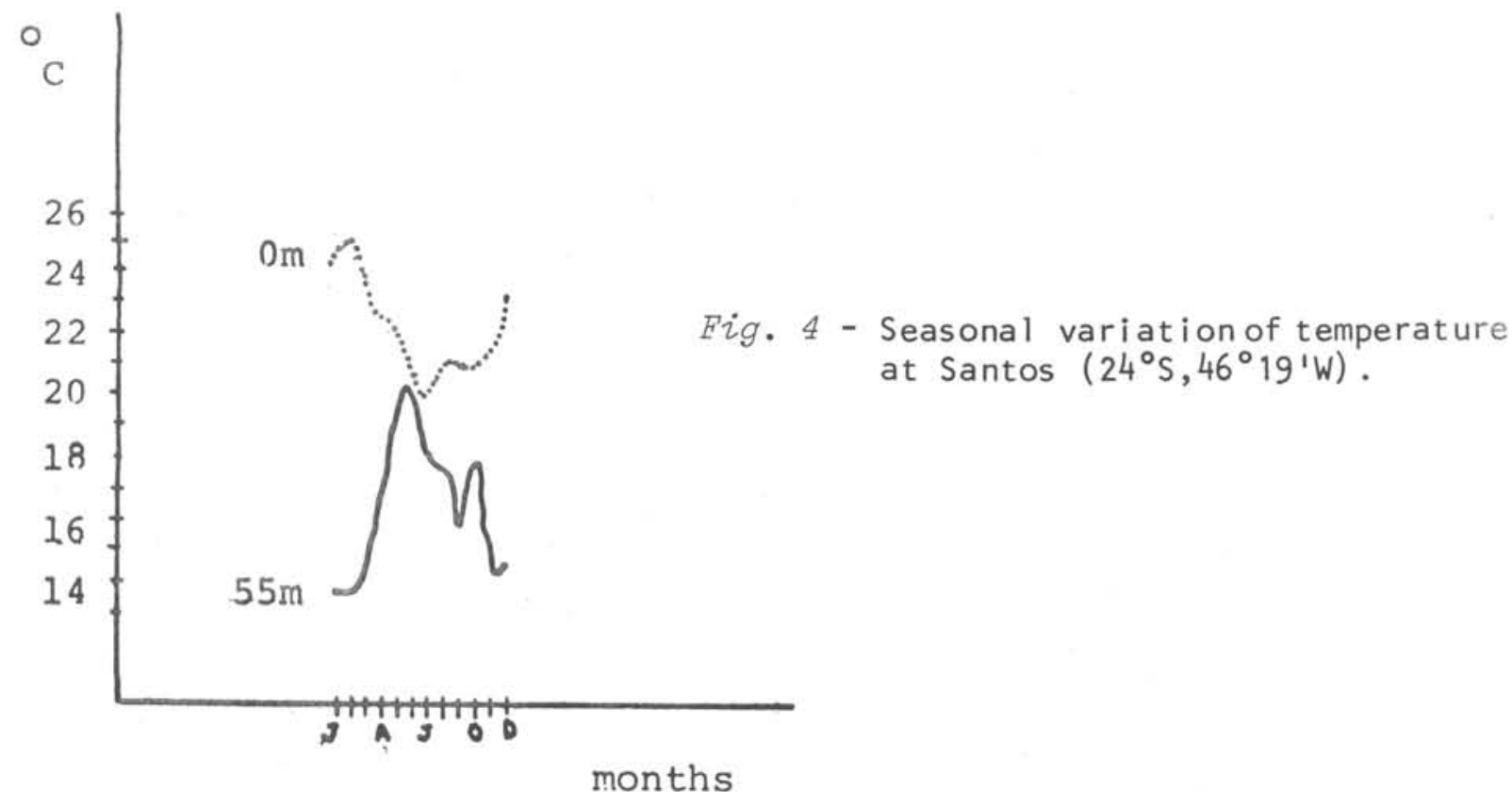

Four years of data were taken for analysis and each year was considered as one realization of the seasonal process. Deviations $\alpha_{j}$ where also calculated from Table I. The 12 mean values of each month, used to calculate the amplitudes $\mathrm{A}_{\mathrm{j}}$ according to (2.37), show the main features of Figure 4, namely a seasonal thermal inversion between $0 \mathrm{~m}$ and $55 \mathrm{~m}$ depths annual variation.

The other effect (Table I) is that the bottom waters have a greater annual amplitude $A_{j}$ of variation than the ones at surface. Amplitude is a minimum at $15 \mathrm{~m}$ with a greater deviation $\gamma_{j}$, indicating the mean annual positioning of the thermocline and that it is a separating line between surface and bottom processes .

Close to the continental boarders the tidal currents are characterized by a great deal of vertical homogeneity of temperature. Annual variation of 
those homogeneous values of temperature are in some respect the response to the variation of the climatic winter-summer thermal values and homogeneity is determined by generally strong tidal currents.

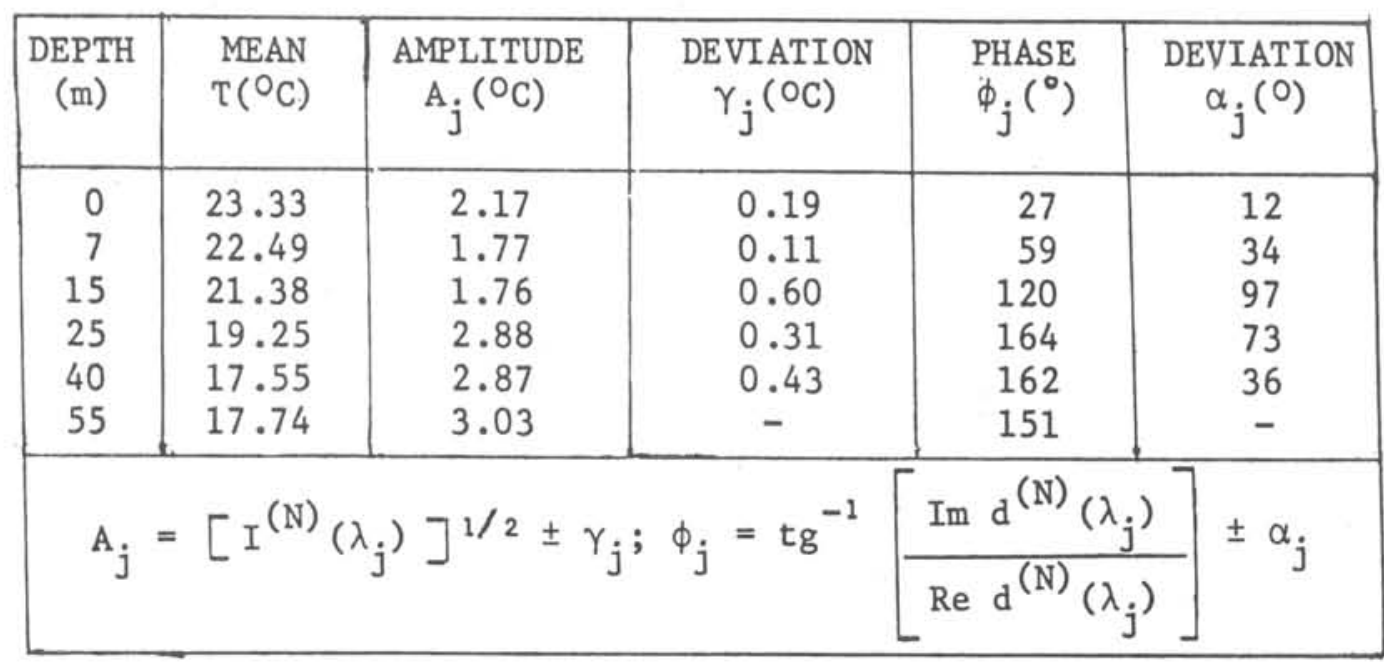

TABLE I - Seasonal variation of temperature at Santos $\left(24^{\circ} \mathrm{S}, 46^{\circ} 19^{\prime} \mathrm{W}\right)$

The equation of motion may be written for such currents as

$$
\left(\frac{d}{d t}-U \cdot \nabla^{2}\right)(u, w)=-\left(\frac{\partial}{\partial x}-\frac{\partial}{\partial z}\right)\left(\frac{p}{\rho}-g z\right),
$$

where $(u, w)$ are the components of current in $x$ and $z$ directions, $p$ is the pressure, $\rho$ the water density, $g$ the gravity and $U$ the turbulent eddy coefficient. Differentiating the first member of (4.1) with respect to $z$ and the second with respect to $\mathrm{x}$ and subtracting we obtain

$$
\left(\frac{d}{d t}-u \cdot \nabla^{2}\right)\left(\frac{\partial u}{d z}-\frac{\partial w}{\partial x}\right)=0
$$

The shear stress and the heat flow connected with flows of the boundary type can be related by

$$
\mathrm{du}=-\frac{\varepsilon_{\mathrm{q}} \cdot{ }^{\mathrm{c}} \mathrm{p} \cdot \sigma}{\mathrm{u}_{\mathrm{z}}{ }^{\mathrm{q}}} \cdot \mathrm{dT},
$$

where $q$ is the amount of heat, $\sigma$ the shear stress, $c_{p}$ the specificheat of water, $\varepsilon_{q}$ the turbulent diffusivity of heat and $T$ is temperature (Eckert \& 
Drake, 1959). Similar expression can be written for dw and dT. It follows from (4.2) and (4.3), after neglecting the advective terms, the contribution in the $\mathrm{x}$ direction that:

$$
k\left(\frac{\partial T}{\partial t}-U_{z} \nabla^{2} T\right)=0,
$$

where $\mathrm{K}$ is the constant appearing in (4.3) for the $\mathrm{z}$ direction. One solution of $(4.4)$ is the diffusion equation, which when solved for $T=T \cdot \cos (\lambda t)$ can be expressed in terms of Fourier components in the form

$$
T(z, t)=T_{0}+\underset{j=1}{2 \sum_{j}} A_{j} e^{-\alpha z} \cdot \cos \left(\lambda_{i} t-\alpha z\right),
$$

where $\alpha=\left(\lambda_{j} / 2 U_{z}\right)^{1 / 2}$ and $\nu_{z}$ the vertical component of $\nu$. For $\lambda_{1}=\frac{2 \pi}{12}, A_{1}$ is the amplitude of the annual component and the first-order term of (4.5) is an exponentially damped wave with increasing depht $\mathrm{z}$, and a corresponding retarding of the phase angle.

Table II shows the results of applying the first term of (4.5) to 12monthly averaged values of temperature resulting from a four-years of data collection at the shelf area of Cananeia (Lat. $25^{\circ} 2$ 's, Long. $048^{\circ} \mathrm{W}$ ).

\begin{tabular}{|c|c|c|c|c|c|c|}
\hline \multirow{2}{*}{$\begin{array}{l}\text { DEPTH } \\
\text { (m) }\end{array}$} & \multicolumn{2}{|c|}{ STATION 1} & \multicolumn{2}{|c|}{ STATION 2} & \multicolumn{2}{|c|}{ STATION 3} \\
\hline & Ampl. & Phase & Ampl. & Phase & Ampl. & Phase \\
\hline $0-7$ & 10.5 & 13.4 & 6.5 & 21.5 & 24.4 & - \\
\hline $7-15$ & 2.4 & 10.6 & 1.2 & 11.6 & 1.3 & 10.9 \\
\hline $15-25$ & - & - & - & - & 1.1 & 7.7 \\
\hline MEAN & 6.4 & 12.0 & 3.8 & 16.5 & 8.9 & 9.3 \\
\hline \multicolumn{7}{|c|}{$\begin{array}{l}\text { Stations } 1,2 \text { and } 3 \text { were located } 5 \text { miles apart in the } \\
\text { shelf area near the town of Cananeia }\left(25^{\circ} 01^{\prime \prime} \mathrm{S} ; 47055^{\prime} \mathrm{W}\right) \text {. }\end{array}$} \\
\hline
\end{tabular}

$$
\text { Values of } \nu_{z}\left(\mathrm{~cm}^{2} / \mathrm{sec}\right)
$$

$$
\text { TABLE II - Values of } \nu_{z}\left(\mathrm{~cm}^{2} / \mathrm{sec}\right)
$$


Values of $U_{z}$ were determined from the damping of $A_{1}$ (amplitude) and the retarding of the annual seasonal cycle (Mesquita \& Morettin, 1976), are similar to those of Crawford (1977) determined for the equatorial waters.

\section{EQUATORIAL OSCILLATIONS}

Meanders of the equatorial waters have recently been reported by Düing et al., 1975. They have $2,600 \mathrm{~km}$ wave lenght and propagate to the west with a phase speed of $1.9 \mathrm{~m} / \mathrm{sec}$. Periodic variation of the field of mass has also been found to follow the patterns of a seasonal variation (Katz etal., 1977).

Temperature and salinity values of these waters were extensively obtained during GATE (GARP Atlantic Tropical Experiment) from July to September, 1974, which showed to be important for the detection of the basic oscillations of the equatorial waters.

Oscillations are believed to be due to atmospheric forcing (Philander, 1976) and they may occur in the meridional or latitudinal sense.

Determination of the main modes of vibration of the water masses are the basic aim of spectral analysis in this case, as no complete theoretical hints are yet available.

Thermal variability for the entire observational period of GATE (JuneSeptember 1974) is shown in Figure 5, for the geographical points $\left(00^{\circ}, 035^{\circ} \mathrm{W}\right)$, $\left(02^{\circ} \mathrm{N}, 038^{\circ} \mathrm{W}\right)$ and $\left(02^{\circ} \mathrm{N}, 035^{\circ} \mathrm{W}\right)$ (Mesquita et al., 1977a).

Four layers of distinct thermal variability can be observed: 1ayer 1 $(0-30 \mathrm{~m})$ associated with currents flowing approximately East to West; 1ayer a $(80-150 \mathrm{~m})$, associated with currents from West to East; $3^{\text {rd }}$ layer $(150-$ $300 \mathrm{~m})$ and $4^{\text {th }}$ layer $(500-1,000 \mathrm{~m})$. 

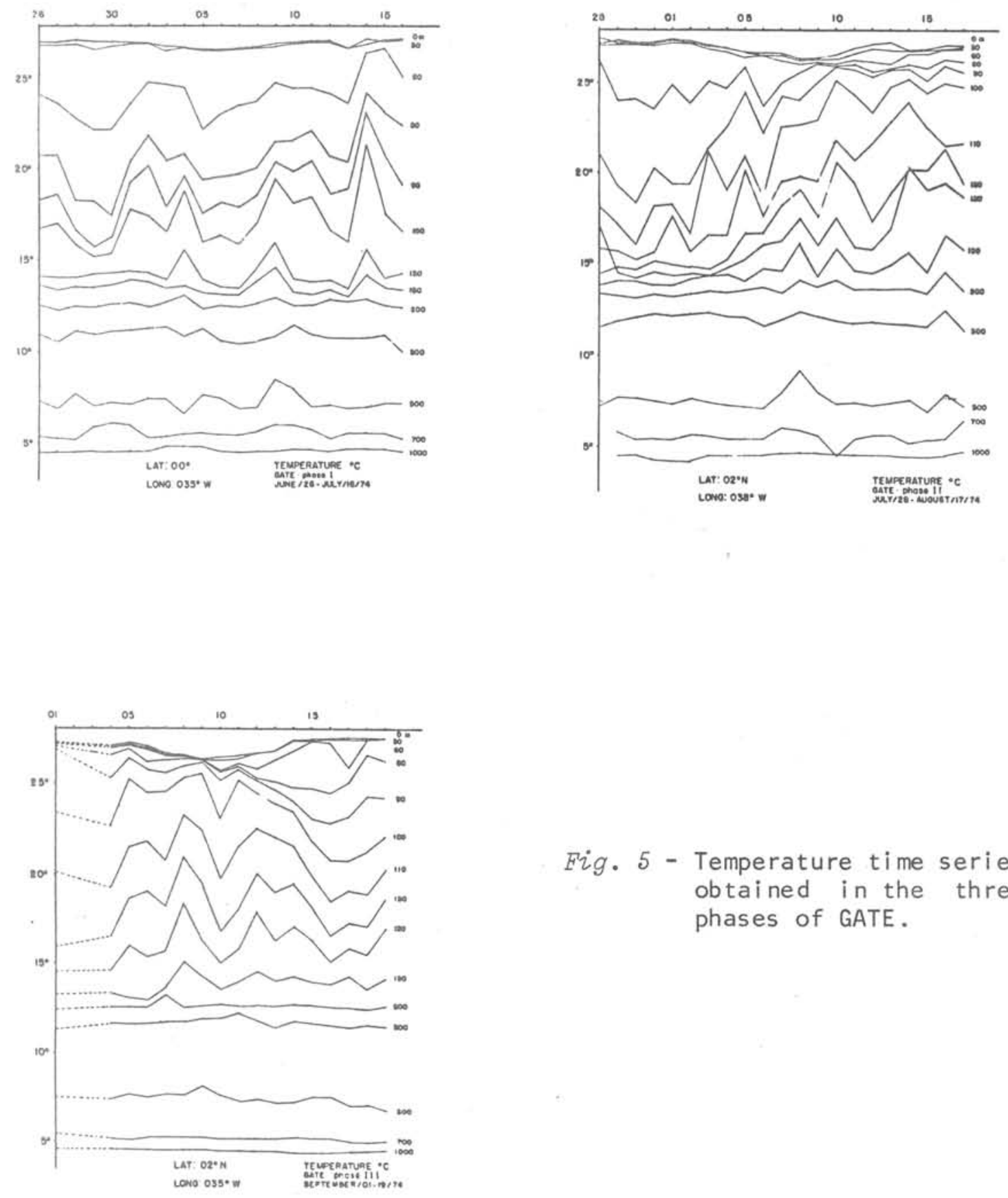

Fig. 5 - Temperature time series obtained in the three phases of GATE.

Series of the $4^{\text {th }}$ layer were analysed using estimators of type (2.42), the small number of observational points being a limiting factor to the a- 
nalysis, but reasonable estimates of the periodicities were obtained as shown in Figure 6. A special program was used and the numbers of lags for covariance estimates are indicated in each spectrum. See discussion on the maximum entropy method concerning the availability of few observations.

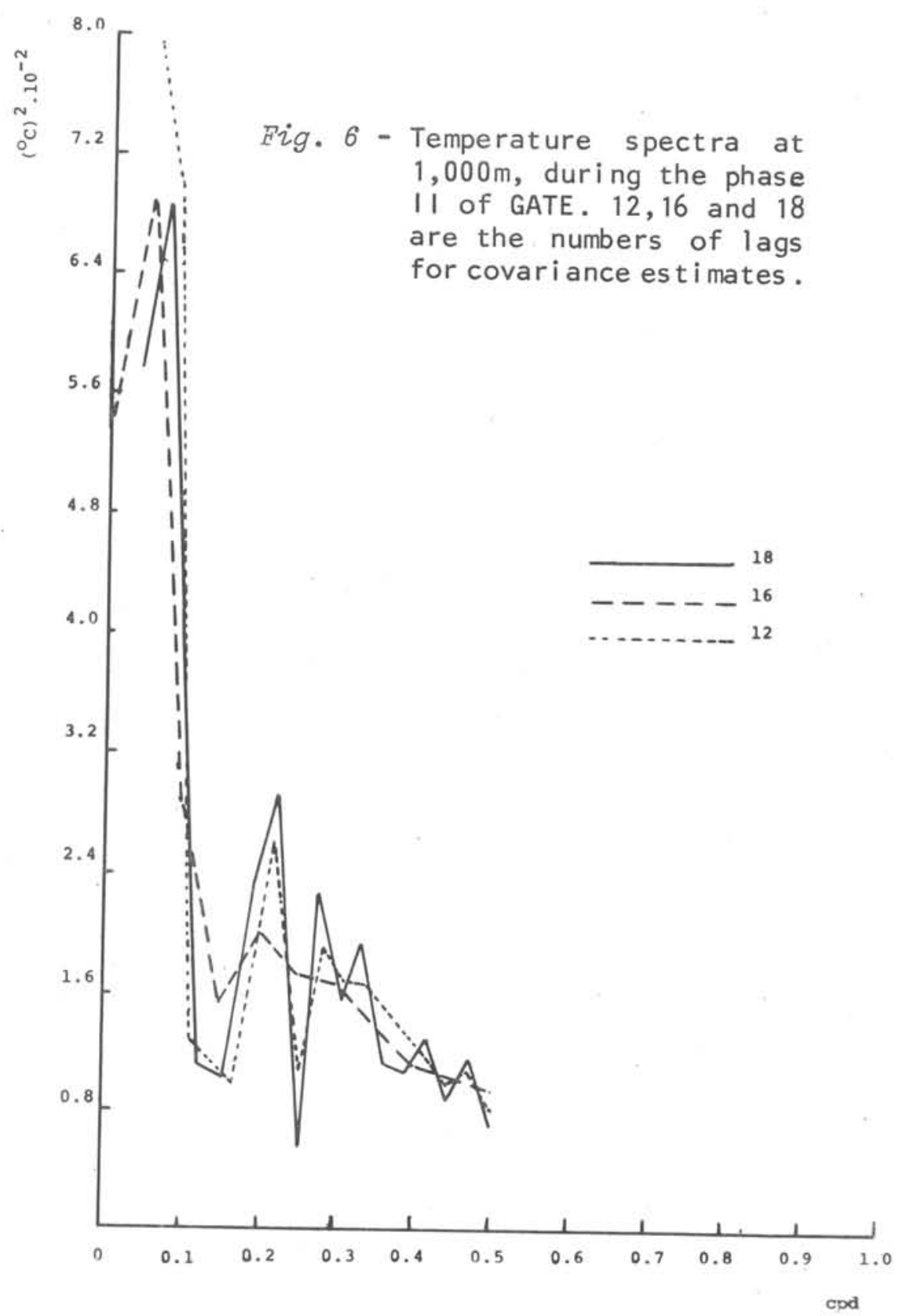


Table III shows the most predominant periods in the series of Figure 5, analysed with (2.35), from what we can see the great range of variability that the equatorial waters undergo. Causes of these variations although believed to be due to atmospheric forcing are still to be proved.

TABLE III - Periods of the thermal fluctuations of the equatorial area shown in figure 5

\begin{tabular}{|c|c|c|c|c|}
\hline \multirow{2}{*}{$\begin{array}{l}\text { DEPTH } \\
(\mathrm{m})\end{array}$} & PHASE I & PHASE II & PHASE III & \multirow{2}{*}{$\begin{array}{l}\text { Most probable } \\
\text { period ranges (days) }\end{array}$} \\
\hline & Periods (days) & Periods (days) & Periods (days) & \\
\hline 0 & 4.2 & $4.5 \quad 2.3$ & 4.2 & $4.2-4.5 ; 2.3$ \\
\hline 60 & 3.52 .3 & $74.2 \quad 2.6$ & 5.6 & $7 ; 4.2-5.6 ; 2.3-2.6$ \\
\hline 100 & 4.2 & 73.5 & 2.8 & $7 ; 3.5-4.2 ; 2.8$ \\
\hline 150 & 2.6 & 3.0 & 5.6 & $7 ; \quad 5.6 ; 2.6-3.0$ \\
\hline 200 & 10.5 & 3.5 & 4.2 & $7-10.5 ; 3.5-4.2$ \\
\hline 500 & 10.5 & 3.0 & $5.6 \quad 2.8$ & $7-10.5 ; 4.2-5.6 ; 2.8-3.0$ \\
\hline 1.000 & $10.55,2$ & 2.8 & 4.2 & $10-10.5 ; 4 \cdot 2-5.2 ; 2.8$ \\
\hline
\end{tabular}

The low frequencies of the spectra were not taken into account, al though by visual inspection they appear to be existent. Periods in each line are distributed in a decreasing order of magnitude and separated to show approximately the different behaviour of the layers 1,2,3 and 4 above refered.

The basic difficulty of oceanic sampling in time series is the short lenght of the series produced relative to the time scales of the processes involved. A method for series with few sampling points is summarized in next section. Other methods for vectorial series are also mentioned.

\section{MAXIMUM ENTROPY（ME） METHOD}

This method is based on the use of an auto-regressive filter applied to the original data. It is known that most of time series encountered can be fitted by an auto-regressive model and the problem that remains is the determination of the order of this model. After that, the spectrum can be easily computed through the Yule-Walker equations relating the coefficients of the filter and the auto-covariances of the process. See Gersh, 1970 and Parzen, 1972 for details. To determine the order $p$ of the process we can plot the innovation variance against $\mathrm{p}$ and see when it stabilizes or use the FPE scheme due to Akaike, 1969a. The FPE (final prediction error) is 
defined to be the expected variance of the prediction error when an autoregressive model fitted to the present series of $X(t)$ is applied to another independent realization of $\mathrm{X}(\mathrm{t})$ to make a one step prediction. See also Akaike, $1969 b$ and 1970 .

It seems that this method is appropriate when we have short records and it has the ability to resolve spectral peaks better than the other methods. Barber \& Taylor, 1977, compare the ME method with the least-squares method and the smoothing periodogram procedure. For other references on the applications of this method to oceanography see U1rych \& Bishop, 1975 and Chen \& Stegen, 1974 .

\section{ROTARY COMPONENTS}

The rotary components method has been applied mainly for analysing current vector time series. The horizontal velocity vector $\underset{\sim}{u}(t)$ can be decomposed into a zonal component $u_{1}(t)$ and a meridional component $u_{2}(t)$. These are assumed to be continuous, stationary stochastic processes with mean zero. It is known that the coherence is invariant under the application of a linear filter to each series, but it is not invariant under coordinate rotation. In order to accomplish this, we decompose the velocity vector, for each frequency, into two counter-rotating circular motions, each with its own amplitude and phase. See Mooers, 1975.

If $\mathrm{f}_{\mathrm{u}_{1} \mathrm{u}_{1}}(\lambda)$ and $\mathrm{f}_{\mathrm{u}_{2} \mathrm{u}_{2}}(\lambda)$ are the auto-spectra of $\mathrm{u}_{1}(\mathrm{t})$ and $\mathrm{u}_{2}(\mathrm{t})$, respectively, then it can be shown that the anticlockwise spectrum is given by

$$
f_{-}(\lambda)=\frac{1}{8} \cdot\left\{f_{u_{1} u_{1}}(\lambda)+f_{u_{2} u_{2}}(\lambda)-2 q_{u_{1} u_{2}}(\lambda)\right\},
$$

and the clockwise spectrum is given by

$$
\mathrm{f}_{+}(\lambda)=\frac{1}{8} \cdot\left\{\mathrm{f}_{\mathrm{u}_{1} \mathrm{u}_{1}}(\lambda)+\mathrm{f}_{\mathrm{u}_{2} \mathrm{u}_{2}}(\lambda)+2 \mathrm{q}_{\mathrm{u}_{1} \mathrm{u}_{2}}(\lambda)\right\},
$$


$\mathrm{q}_{\mathrm{u}_{1} \mathrm{u}_{2}}(\lambda)$ being the quad-spectrum between $\mathrm{u}_{1}(t)$ and $\mathrm{u}_{2}(t)$. The total spectrum of kinetic energy is given by

$$
f_{t}(\lambda)=f_{-}(\lambda)+f_{+}(\lambda) \text {. }
$$

The amplitudes of these rotary spectra are independent of the orientation of the coordinate system where the process is described. For applications see Gonela, 1972. The case of rotary bi-spectra is considered by Yao et al., 1975 , to analyse the non-Gaussian nature of a vector random process.

Figure 7 shows the rotary spectra of currents at $25^{\circ} \mathrm{S} 045^{\circ} \mathrm{W}$. Currents at the area show periodicities of 3-6 days, diurnals and semidiurnals and flow with a pronounced anti-hourly sense (Mesquita et al., 1977b).

Fig. 7 - The rotary spectra of currents - $40 \mathrm{~m}$.

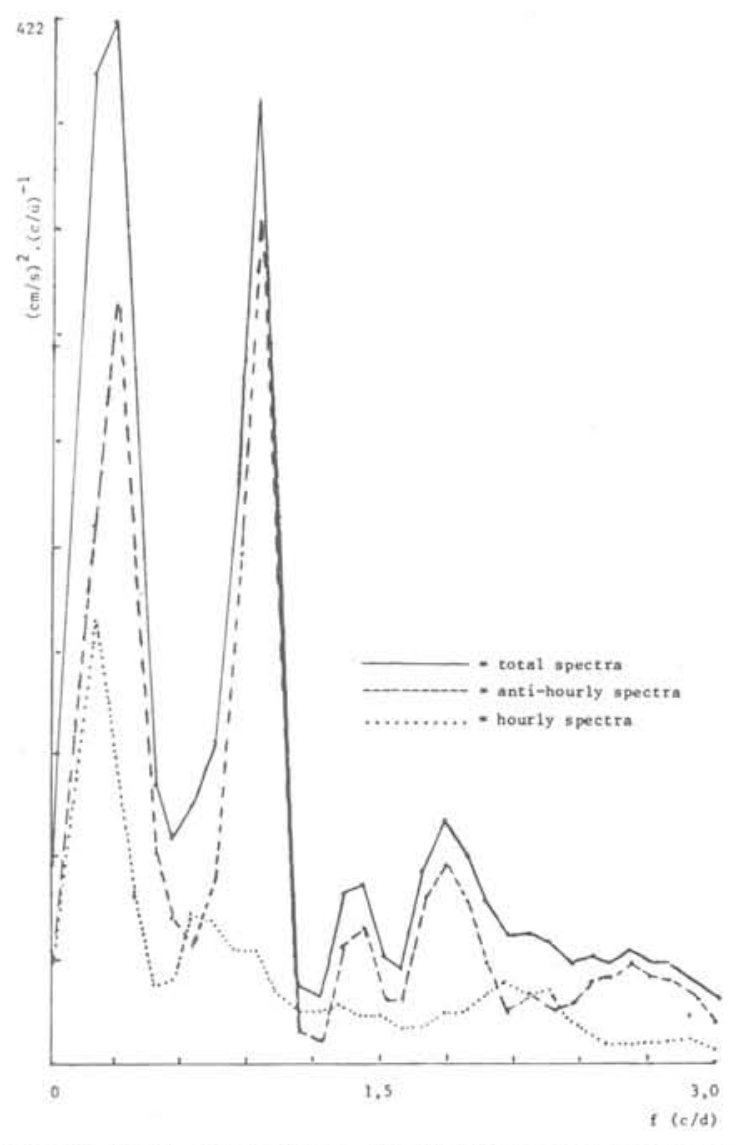

The full, dashed, and pointed limes are respectively related to the total, anti-hourly, and fourly stectra. 


\section{B IBLIOGRAPHY}

AKAIKE, H. 1969a. Fitting auto-regressive models for prediction. In: Rosenblatt, M. \& Van Atta, C., ed. - Statistical models and turbulence. New York, Springer-Verlag.

1969b. Power spectrum estimation through auto-regressive model fitting. Ann. Inst. statist. Math., Tokyo, 21:407-419.

1970. Statistical predictor identification. Ann. Inst. statist. Math., Tokyo, 22:203-217.

BARBER, F. G. \& TAYLOR, J. 1977. A note on free oscillations of Chedabucto Bay. Manuscr. Rep. Ser., Mar. Sci. Directorate, Ottawa, (47).

BRILLINGER, D. R. 1965. An introduction to polyspectra. Ann. math . Statist., 36(5):1351-1373.

1970. The identification of polynomial systems by means of higher order spectra. J. Sound Vibr., 12(3):301-313.

York, Holt, Rinehart \& Winston.

\& ROSENBLATT, M. 1967a. Asymptotic theory of estimates of $\mathrm{K}$-th order spectra. In: Harris, B., ed. - Spectral analysis of time series. New York, Wiley \& Sons, p. 153-188.

1967b. Computation \& interpretation of K-th order spectra. In: Harris, B., ed. - Spectral analysis of time series. New York, Wiley \& Sons, p. 189-231.

CARTWRIGHT, D. E. 1971. Some ocean tide measurements of the Eighteenth Century, and their relevance today. Proc. R. Soc., ser. B, 72(32):331-339.

\& EDDEN, A. C.

1973.

harmonics. Geophys. J. R. astr. Soc., 33:253-264.

\& TAYLER, R. J.

1971. New computations of the tide generating potential. Geophys. J. R. astr. Soc., 23:45-74.

CHEN, W. Y. \& STEGEN, G. R. 1974. Experiments with maximum entropy power of sinusoids. J. geophys. Res., 79:3019-3022.

COHEN, A. \& JONES, R. H. 1969. Regression on a random field. J. Am. statist. Ass., 64:1172-1182.

CRAWFORD, W. R. 1976. Turbulent energy dissipation in the Atlantic Equatorial undercurrent. PhD These. University of British Columbia, Oceanographic Institute. 
DOODSON, A. T. 1921. The harmonic development of the tide generating potential. Proc. R. Soc., ser. A, 100:305-328.

1957. The analysis and prediction of tides in shallow waters. Int. hydrogr. Rev., 33:85-126.

DUING, W.; HIZARD, P.; KATZ, E.; MEINCKE, J.; MILLER, L.; MOROSHKIN, K. . ; PHILANDER, G.; RYBNIKOV, A. A.; VOIGT, K. \& WEISBERG, R. 1975. Meanders and long waves in the Equatorial Atlantic. Nature, Lond .; 257(5524).

ECKERT, E. R. \& DRAKE, R. M. 1959. Heat and mass transfer. New York, MacGraw-Hill.

FRANCO, A. dos S. 1964. Harmonic analysis of tides for 7 days of hourly observations. Int. hydrogr. Rev., 41(2):109-142.

\& ROCK, N. J. 1971. The fast Fourier transform and its applications to tidal oscillations. Bolm Inst. oceanogr., S Paulo, $20: 145-199$.

GASSER, T. 1972 .

System identification, polyspectra and related functions. Dissertation (Doctor of Math.). Swiss Federal Institute of Technology.

GERSH, W. 1970. Spectral analysis of EEG's by auto-regressive decomposition of time series. Math. Biosc., 7:205-222.

GODFREY, M. D. 1965. An exploratory study of the bispectrum of economic time series. Appl. Statist., 14(1):48-69.

GODIN, G. 1972. The analysis of tides. Liverpool, Liverpool University Press, 264p.

GONELLA, J. $\quad 1972$. A rotary-component method for analysing meteorological and oceanographic vector time series. Deep Sea Res., 19:833-846.

HANNAN, E. J. 1966. Spectral analysis for geophysical data. J. R. astr. Soc., $11: 225-236$.

HORN, W. 1960. Some recent approaches to tidal problems. Int. hydrogr. Rev., 37(2):65-68.

JENKINS, G. M. \& WATTS, D. G. 1968. Spectral analysis. Holden-Days, $535 \mathrm{p}$.

JONES, R. H. $\quad$ 1963. Stochastic processes on a sphere as applied to meteorological 500-milibar forecasts. In: Rosenblatt, M., ed. - SIAM Proc, of Time Series Analysis. New York, John Wiley, p. 119-124.

KATZ, E.; BELEVITSCH, R.; BRUCE, J.; BUBNOV, V.; COCHRANE, J.; DUING, W.; HIZARD, P.; LASS, H. V.; MESQUITA, A. R. de; MILLER, L. \& RYBNIKOV, A. A. 1977. Zonal pressure gradient along the Equatorial Atlantic. J. mar. Res., 35(2):203-307. 
KOOPMANS, L. H. 1974. The spectral analysis of time series. New York, Academic Press.

MESQUITA, A. R. de; MAGLIOCCA, A. \& MBROSIO Jr., 0. 1977a. Physical and chemical variability at $35-40^{\circ} \mathrm{W}$ of the Equatorial Atlantic upper layers . Re1. int. Inst. oceanogr., Univ. S Paulo, (8):1-27.

\& MORETTIN, P. A. 1976. "Aplicações da anālise espectral à oceanografia" (in Portuguese). 2nr Brazilian Symp. on Prob. and Statistics, (in press).

FESTA, M. \& LEITE, J. B. A. bilidade do campo de massa (Ponto: 25 S; 46 W). Re1. Cruzeiros, sér. N/Oc. "Prof.W. Besnard", Inst. oceanogr. Univ. S Paulo, (3):1-27.

MEUS, J. 1962. Tables of moon and sun. Belgium, Kessleberg Sterrenwacht.

MOEERS, C. N. K. 1973. A technique for the cross spectrum analysis of pairs of complex-valued time series, with emphasis on properties of polarized components and rotational invariants. Deep Sea Res., 20:11291141.

MUNK, W. \& CARTWRIGHT, D. E. 1966. Tidal spectroscopy and prediction. Phil. Trans. R. Soc., ser. A, 259:533-581.

\& HASSELMANN, K. 1964. Super-resolution of tides. In: Yoshida,
K., ed. - Studies on oceanography. Tokyo, University of Tokyo Press, p.
339-344.

PARZEN, E. 1972. Some recent advances in the time series analysis. In: Rosenblatt, M. \& Van Atta, C., ed. - Statistical models and turbulence. New York, Springer-Verlag.

PHILANDER, G. 1976. Instabilities of zonal equatorial currents. J. geophys. Res., 81(21):3725-3735.

ROY, R. 1976. Spectral analysis for a random process on the sphere. Ann. Inst. statist. Math., Tokyo, 28(1):91-99.

TUKEY, J. W. 1961. Discussion, emphasizing the connection between analysis of variance and spectrum analysis. Technometrics, 3:1-29. 
ULRYCH, T. J. \& BISHOP, T. N. 1975. Maximum entropy spectral analysis and auto-regressive decomposition. Rev. geophys. space Phys., 13:183-200.

YAO, N.-C.; NESHYBA, S. \& CREW, H. 1975. Rotary cross-bispectra and energy transfer functions between non-gaussian vector processes.I. Development and example. J. phys. Oceanogr., 5(1):164-172.

YAGLOW, A. M. 1961. Second-order homogeneous random fields. Proc. Berkekey Symp. math. Statist. Probab., 4th, 2:593-622.

(Received November 18/1977) 